\title{
Effect of Cavity Flow on Landing Gear Aerodynamic Loads
}

\author{
Utsav Oza ${ }^{*}$ Zhiwei $\mathrm{Hu}^{\dagger}$ and Xin Zhang* \\ Faculty of Engineering and the Environment, University of Southampton, Southampton, SO16 7QF, UK
}

Delayed Detached Eddy Simulations (DDES) were performed on a scaled model of a generic aircraft Nose Landing Gear (NLG). The model includes medium and large sized NLG components which replicates the original geometry. This work is performed as part of the ALGAAP (Advanced Landing Gear Aero-loads and Aero-noise Prediction) project supported by UK TSB (now called Innovate UK). Simulations were performed on three different configurations with the fuselage included to investigate the effects of the landing gear bay cavity on aerodynamic loads. The three configurations are - (1) a fully extended NLG with a sealed cavity, (2) a fully extended NLG with the front doors closed and a partially exposed cavity and (3) a fully extended NLG with the front doors open and a fully exposed cavity. The leg (rear) doors are deployed for all configurations. Good agreement with experimental data is achieved, with the relative errors of drag and moment coefficients less than $3 \%$. The interior of the cavity has some geometrical features, the complex flow pattern within the cavity and its effect on the surrounding NLG components are investigated. For the NLG with a partially exposed cavity, an open cavity flow is observed possessing periodic pressure fluctuations whose frequency are in good agreement with Rossiter modes. In contrast, due to the geometry shape and complexity, a typical open cavity flow was not observed for the NLG with a fully exposed cavity in spite of a low length to depth ratio $(L / D \sim 2.4)$ of the cavity.

\section{Nomenclature}
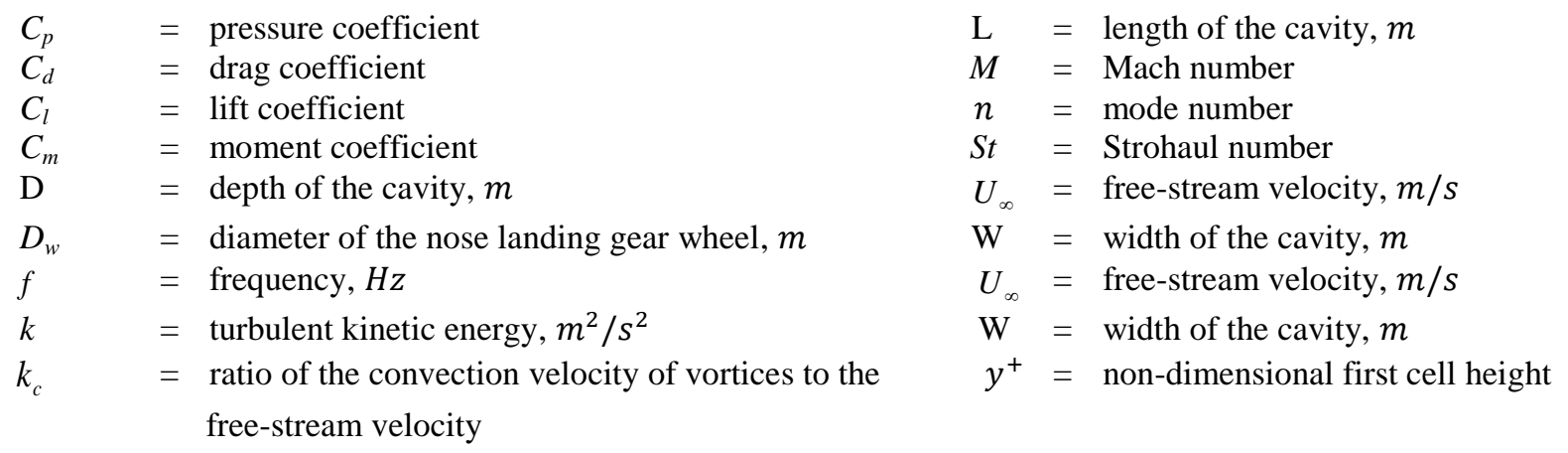

Greek symbols

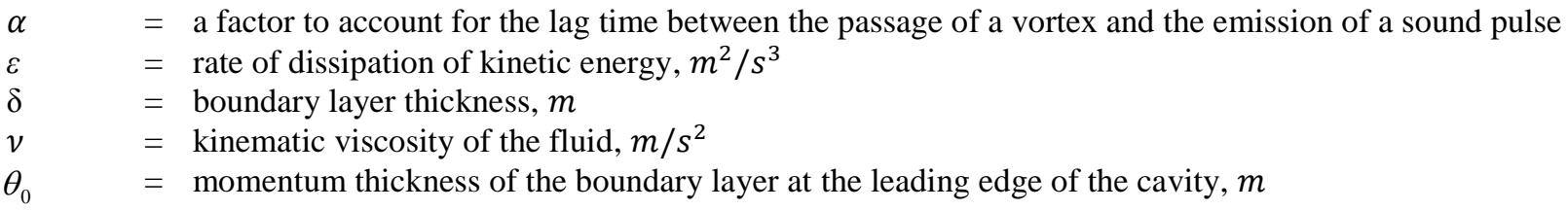

\footnotetext{
${ }^{*}$ PhD student, Airbus Noise Technology Centre, Email: u.oza@soton.ac.uk, AIAA Member.

† Lecturer, Airbus Noise Technology Centre, Email: z.hu@ soton.ac.uk, AIAA Member.

* Airbus Noise Technology Centre. Also Chair Professor, Department of Mechanical and Aerospace Engineering, The Hong Kong University of Science and Technology, Clear Water Bar, Kowloon, Hong Kong SAR, China. Associated fellow AIAA.
} 


\section{Introduction}

$\mathrm{F}$ undamental research of cavity flow, in the field of aeronautics, was mostly targeted towards better understanding of the flow physics around components such as aircraft weapon bays and undercarriage cavities. Investigations of unsteady aerodynamic loads and acoustic effects caused by the cavity flow began in the 1950's. Apart from the highly separated flow due to landing gear components, an exposed undercarriage cavity also contributes to the unsteady flow features. The complex nature of the flow within the cavity generates aerodynamic loads, pressure fluctuations and acoustic radiation. Even though the problem has been known for a long time, effective control of oscillations in the cavity is still a challenging task. Cavity has the ability to disrupt the free-stream flow underneath the aircraft fuselage. Hence, the presence of a cavity can significantly change the flow field and create additional unsteady aerodynamic loads on surrounding components (landing gear doors, struts, drag braces, etc.).

During the 1960's, Rossiter ${ }^{1}$ performed an extensive study of cavity flow at subsonic and transonic speeds which provided a detailed description of the problem. It was observed that pressure waves were formed due to the interaction of the rear cavity wall with the large scale vortices from the shear layer. The pressure waves then travel upstream and further interact with the shear layer. Due to this interaction, the shear layer oscillates and creates a feedback loop which results in a self-sustained system. The noise generated due to this phenomenon has also gained attention from researchers due to the need for a quieter aircraft ${ }^{2,3}$.

A cavity can be identified based on its relative dimensions of length $(L)$, depth $(D)$ and width $(W)$. The ratio of $L / D$ is a non-dimensional number to represent a deep or shallow cavity. Rossiter ${ }^{1}$ mentioned in his work that the cavity can be considered to be deep if $L / D<4$. The $L / W$ ratio refers to the effect on the cavity flow due to the third dimension (width). According to Block ${ }^{4}$ and Ahuja et al. ${ }^{5}$, the flow field can be considered two-dimensional for $L / W$ $<1$. Cavity flow can be classified on the basis of its flow phenomenon. It is mainly categorized into closed, open and transitional flow. In a closed cavity, the flow at the front and the rear wall can be considered as separate flow across a backward and a forward facing step. The flow separates at the cavity leading edge, re-attaches to the base of the cavity and then detaches as it travels towards the trailing edge. While, in an open cavity flow, a shear layer is formed at the cavity leading edge which then interacts with the rear wall of the cavity. The resonance problem related to the cavity flow is generally associated with an open cavity, which usually has $L / D$ less than or equal to 10 . Periodic pressure oscillations are observed in this type of cavity flow.

Rossiter $^{1}$ derived a semi-empirical formula to predict the resonance frequency of an open cavity which is described in equation (1), where $f$ is the frequency, $L$ is the length of the cavity, $U_{\infty}$ is the free-stream velocity, $n$ is the mode number, $M$ is the Mach number, $k_{c}$ is the ratio of the convection velocity of vortices to the free-stream velocity, $\alpha$ is a factor to account for the lag time between the passage of a vortex and the emission of a sound pulse at the trailing edge of the cavity. Rossiter ${ }^{1}$ found that by taking the values of $\alpha=0.25$ and $1 / k_{c}=1.75$ the results of the empirical equation agreed well with his experimental data. The value of $k_{c}$ can vary from 0.3 to 0.6 . Later, Heller et al. ${ }^{6}$ modified this formula to account for the difference in the sound speed inside the cavity and the free stream.

$$
\frac{f L}{U_{\infty}}=\frac{n-\alpha}{M+1 / k_{c}}
$$

Tam and Block ${ }^{7}$ argued that Rossiter's equation cannot accurately predict the resonance frequency over a wide range of Mach number, especially at supersonic speeds. So, they proposed a new mathematical model which incorporated the effect of the finite shear layer thickness and acoustic reflections from the front and the bottom wall of the cavity. However, they agreed that Rossiter's equations can be applied to predict resonance frequency in the range of $0.4 \leq M \leq 1.2$. Block ${ }^{8}$ stated that for a given Mach number, the Strohaul number $(S t)$ increases with increase in $L / D$. In his other work, Block ${ }^{9}$ mentioned that the $S t$ is relatively insensitive to the $L / D$ ratio if it is greater than or equal to 3 . For the test case investigated in this study, the $L / D$ ratio is 2.4 and 1 for the fully and partially exposed cavities respectively (refer to Section II). So, an increase in $S t$ is expected from the unsteady simulation results when the partially exposed cavity is compared with the fully exposed one. Block ${ }^{9}$ also mentioned that depth wise standing waves can be found in the cavity with $L / D \leq 1$, leading to differences in the predicted $S t$.

The boundary layer thickness at the cavity leading edge affects the shear layer oscillation. According to Sarohia ${ }^{10}$, to initiate a self-sustained oscillation in the cavity, a minimum cavity length is required which depends on the freestream velocity, kinematic viscosity of the fluid $(v)$ and the momentum thickness of the boundary layer at the 
leading edge $\left(\theta_{0}\right)$. The frequency of the cavity oscillation increases with the decrease in boundary layer thickness. Hence, for CFD simulations, a resolved boundary layer mesh is required near the leading edge of the cavity. Rubio et al. ${ }^{11}$ performed Large Eddy Simulation (LES) at a low subsonic speed range on a two-dimensional cavity. The study showed three different oscillation regimes in the cavity flow; shear-layer mode, wake mode and mixed mode. Rowley et al. ${ }^{12}$ provided a detailed insight regarding the instabilities in an open cavity flow due to the shear layer mode and the wake mode. In the shear-layer mode, oscillations at Rossiter frequencies occur due to the interaction between the pressure waves emanated from the rear wall and the shear layer vortex shedding. In the wake mode, a significant vortex motion is observed across the cavity centreline. The frequency of the oscillation becomes independent of the Mach number and the feedback mechanism diminishes. The relation between flow oscillations and the cavity drag was investigated by Gharib and Roshko ${ }^{13}$. It was observed that the mean drag coefficient is low when the shear layer is not oscillating or it is in the shear-layer mode. However, the drag increases an order of magnitude when it shifts to the wake mode.

A study relevant to the current research was done by Langtry and Spalart ${ }^{14}$. They performed DES simulations on a NLG cavity with partially retracted gear and closed rear doors. This configuration was selected due to the vibration and unsteady loads observed during the wind tunnel tests. A detailed model representing a long range aircraft was used in their wind tunnel tests. However, the geometry was simplified for DES simulations. The landing gear was represented using simple cylindrical strut and wheel. Also, a half model was used for CFD simulations to reduce the grid size. Multi-block hexahedral meshes were used with 5 and 12 million nodes for coarse and fine mesh respectively. Mach contour plots from the simulation showed the oscillation of shear layer due to pressure waves.

The two main aspects of the ALGAAP project are wind tunnel tests of a scaled aircraft landing gear model and its validation using CFD simulations. The wind tunnel model consists of medium and large size components on the NLG and the fuselage. Results obtained from CFD simulations are discussed in this paper and compared with experimental data. The current research intends to study the change in the flow features and unsteady aerodynamic loads on complex landing gear components. This topic is studied by comparing three configurations- Case-1: a fully extended NLG with a sealed cavity, Case-2: a fully extended NLG with a partially exposed cavity and Case-3: a fully extended NLG with an exposed cavity. Section II introduces the test configuration used for wind tunnel experiments, followed by Section III which reviews the mesh generation and CFD strategy. In Section IV, simulation results are discussed. Finally, a conclusion is presented in Section V.

\section{Test configuration}

Landing gears have evolved into a very complex system with several mechanical, hydraulic and electrical systems embedded within and around a landing gear. The geometry for the current CFD model contains key components of a NLG except hydraulic pipes, electrical cables and very small details, representing the wind tunnel model shown in Figure 1. It consists of a fuselage and major parts of the NLG which closely represent that of a long range aircraft. In addition, the front and rear doors are also incorporated in the geometry. Although, the NLG geometry is not as detailed as a realistic landing gear, the present case is geometrically complex when compared to previous configurations which were utilized to study cavity flow ${ }^{15}$. A number of previous studies involving simulation of complex NLG were found in the literature ${ }^{16-23}$. However, in those studies, cavity flow associated with the NLG was not considered. In most of the cases, the cavity was either sealed or a flat plate was used on top of the NLG for the CFD simulations. Thus the effect of the cavity on flow over complex configurations has not been thoroughly investigated. Thus, this study is a step forward towards better understanding of the complicated cavity flow and its aerodynamic effect on surrounding parts.

The landing gear undercarriage cavity of civil aircrafts generally falls under the category of open cavity flow. A detailed review of literature ${ }^{15}$ showed that all previous studies were performed using a clean cavity configuration or a simplified version of an aircraft cavity. In the literature, the shape of the cavity is assumed to be rectangular with the inner surface parallel to the free stream flow. The current cavity case represents that of a realistic aircraft landing gear bay and the inner surface is angled with respect to the free-stream. The $L / D$ ratio of the cavity is 2.4 when the full cavity is fully exposed (both front and rear doors open). The $L / D$ ratio of the cavity decreases to about 1 when the front doors are closed and the rear doors are kept open. These low $L / D$ ratios represent a deep cavity and open flow. The $L / W$ ratio is close to 2 for the fully exposed cavity. In addition, other geometrical features and landing gear parts are present within the cavity which makes it an interesting case to study. The CFD configuration with divided surface domains used for Case-3 is presented in Figure 2. The inflow is in the $x$ direction. Front and rear 
doors are also present, but not shown in the figure for the sake of visibility of other components. All three configurations, (1) Case-1: a fully extended landing gear with a sealed cavity, (2) Case-2: a fully extended landing gear with a partially exposed cavity and (3) Case-3: a fully extended landing gear with a fully exposed cavity, are presented in Figure 3. It should be noted that the rear doors are present in all three configurations, while the front doors are present only in Case-3.

\section{Mesh generation and simulation}

To reduce the complexity of the CFD model, some small parts (such as light clusters) were removed from the wind tunnel model to create a simplified yet realistic model. Wind tunnel data for this simplified model was separately obtained to validate CFD results. In the past, simulations had been performed (on the same model) using unstructured tetrahedral $^{24}$ and hybrid ${ }^{25}$ (structured + unstructured) meshes. Previous simulations with tetrahedral mesh did not provide sufficient accuracy which led to a case study of hybrid meshing strategies to observe its effect on results. The simulation performed using a hybrid mesh showed a better prediction of the mean aerodynamic loads. However, this improvement in accuracy came at a cost of significant increase in the mesh generation time. So, it was difficult to implement this strategy to perform simulations on different geometrical configurations of the same model. Hence, focus is concentrated on using an unstructured mesh generator with hexahedral cells to benefit from higher order cell type $\mathrm{e}^{26,27}$.

Compared to structured meshes, an unstructured mesh suffers from some disadvantages such as excess computational memory, lower cell quality and lower accuracy. On the contrary, a significant amount of manual work and time is saved in mesh generation for complex cases. So, in this case, a commercial package is utilized for efficient mesh generation of complex landing gear configurations. An unstructured mesh requires careful selection of the cell size and shape in order to achieve the optimum performance. An attempt is made to utilize a hexdominant mesh generator with a resolved boundary layer with target $y^{+}$ranging between 1 2.

Using a commercial mesh generator, HEXPRESS ${ }^{\mathrm{TM}} / \mathrm{Hybrid}$, unstructured hex-dominant grids were generated on the three different configurations presented in Figure 3. All configurations included the whole fuselage. The landing gear bay cavity is included for Case- 2 and Case-3. The simulation domain is 2 fuselage lengths upstream, 4 fuselage lengths downstream and 0.5 fuselage lengths on the sides, top \& bottom from the NLG. A velocity inlet boundary condition is applied at the inflow. Pressure outlet boundary condition is used at the outlet of the domain. A no-slip wall condition is applied on all landing gear solid surfaces. A slip wall condition is used on the rest of the domain boundaries surrounding the model. The surface mesh on the NLG is presented in Figure 4. In addition, a cutting plane at the fuselage center is depicted in Figure 5 to visualize the relative cell size and refinement around the NLG. Two refinement zones (zone 1 and 2 as shown in Figure 5) around the NLG have cell size of $2 \%$ of the wheel diameter. Then further downstream the cell size increases to $4 \%$ (zone 3) and $8 \%$ (zone 4) of the wheel diameter. The total cell count for each case is given in Table 1. For Case-2 and Case-3, the meshes are generated following similar strategy and the mesh size is approximately twice compared to Case- 1 due to the addition of cells within the cavity. Table 2 presents the cell refinement size on the surface of the NLG components. Since, the mesh convergence study of such complex cases can be computationally expensive; the refinement parameters were selected by studying CFD simulations in the literature ${ }^{21,28}$ and also, from the previous simulations on the same NLG geometry (with tetrahedral ${ }^{24}$ and hybrid mesh $^{25}$ ). The mesh quality was inspected using 'checkMesh' command available in OpenFOAM®. The mesh passed the quality check with maximum non-orthogonality and cell skewness below 80 and 4 , respectively.

All computations are performed using OpenFOAM® version 2.3.0 on the IRIDIS computing facility at University of Southampton. Firstly, for each case, the realizable $k$ - $\varepsilon$ turbulence model is chosen to perform SRANS simulation ( $2^{\text {nd }}$ order central scheme) for 10,000 iterations. The steady solution is used to initialize the DDES simulation with a Spalart-Allmaras (S-A) turbulence model. The default S-A DDES ${ }^{29}$ model in OpenFOAM® version 2.3.0 is utilized without any transition specification leading to fully turbulent boundary layer ${ }^{30}$. A second order implicit time marching scheme is utilized. The simulation is initially started with a time step of $2.5 \times 10^{-7} s$ during the transient phase when smaller eddies start to form from the SRANS solution. Then the time step is gradually increased to $5 \times 10^{-7} \mathrm{~s}$. The maximum CFL number during the computation was 5 . High CFL numbers were only found near the sharp geometric edges. The computation is performed for a total solution time of $0.08 s$ which corresponds to the flow passing twice through the fuselage length, equivalent to 60 times flow through the 
wheel or 16 times flow through the fully exposed cavity. Pressure probes are placed in the wake region, behind each main component and within the cavity. The data is sampled during the last $0.04 \mathrm{~s}$.

Table 1: Mesh size for NLG configurations.

\begin{tabular}{|l|c|c|c|c|}
\hline \multicolumn{1}{|c|}{ Number } & Cavity & Front Door & Rear Door & Cell count \\
\hline Case-1 & Sealed & Closed & Open & 14.7 million \\
\hline Case-2 & Partially exposed & Closed & Open & 29.6 million \\
\hline Case-3 & Fully Exposed & Open & Open & 32.6 million \\
\hline
\end{tabular}

Table 2: Surface cell size relative to wheel diameter (Case-3).

\begin{tabular}{|l|c|}
\hline \multicolumn{1}{|c|}{ Parameter } & $\begin{array}{c}\text { Case-3 } \\
\text { Current Hex-dominant mesh } \\
\text { (Cell size relative to wheel diameter })\end{array}$ \\
\hline Mesh size & 32.6 million cells \\
\hline Fuselage & $4 \%-8 \%$ \\
\hline Cavity & $2 \%$ \\
\hline Doors & $0.5 \%-1 \%$ \\
\hline Wheels & $2 \%$ \\
\hline Strut and braces & $1 \%$ \\
\hline Steering assembly & $0.5 \%$ \\
\hline Torque link & $0.5 \%$ \\
\hline
\end{tabular}

\section{Simulation results}

\section{A. Comparison with Experimental data}

For validation purposes, the simulation data is compared with the mean drag and pressure data obtained from the wind tunnel test $\mathrm{s}^{31}$ performed at Airbus. The available experimental data for configuration with open doors and a fully exposed cavity are compared to the Case-3 predictions. The comparison of the mean lift $\left(C_{l}\right)$, drag $\left(C_{d}\right)$ and moment ( $C_{m}$ ) coefficients for the NLG is given in Table 3. For $C_{d}$ and $C_{m}$, the prediction error (relative to the wind tunnel data) is approximately $2 \sim 3 \%$. The relative error for $C_{l}$ is $24.1 \%$; however, the magnitude of the lift coefficient is small (approximately $10 \%$ of the $C_{d}$ ). The absolute errors for all the coefficients are in similar order of magnitudes. Further verification of the simulation is performed by comparing the mean pressure data obtained from the wind tunnel tests. Figure 6 shows the pressure coefficient on the port-side wheel centerline. Figure 7 presents comparison of simulation and experimental data at various probe locations on the fuselage and inside the cavity (on the fuselage center-line plane). The pressure coefficient matches very closely in Figure 7, which indicates that the simulation for Case-3 produces correct mean flow features under the fuselage and within the cavity. Figure 8 shows the probe locations (upper and lower rows) on the front and rear door. Figure 9 and Figure 10 presents the pressure coefficient on the probes located on the front and rear doors, respectively. When simulation results are compared to experimental data, a similar profile of pressure change is noticed along the length of the doors; both on the upper and lower rows. Thus, a good agreement of the simulation results with the experimental data is observed. 
Table 3: Force and moment coefficient for the NLG (Case-3).

\begin{tabular}{|l|c|c|c|c|}
\hline \multicolumn{1}{|c|}{ Parameter } & Wind tunnel data & Simulation results & Absolute error & Relative Error \\
\hline Drag coefficient, $C_{d}$ & 1.0950 & 1.0666 & 0.0284 & $2.6 \%$ \\
\hline Lift coefficient, $C_{l}$ & -0.1355 & -0.1028 & 0.0327 & $24.1 \%$ \\
\hline Moment coefficient, $C_{m}$ & -1.8394 & -1.7992 & 0.0402 & $2.2 \%$ \\
\hline
\end{tabular}

\section{B. Mean flow features}

The surface wall $y^{+}$and pressure coefficient contours on the NLG are depicted in Figure 11. During the mesh generation process the first cell height of the resolved boundary layer was first estimated such that $y^{+}$falls in the range of 1 2 in the simulation. The values of wall $y^{+}$contours confirm that the estimated first cell height was appropriate. In Figure 11(b), the front part with high pressure coefficient indicates the NLG components interacting with the undisturbed free-stream flow. It is observed that the maximum pressure coefficient does not occur at the wheel centerline, but towards the inner side of the wheel (towards the strut). The offset is due to the presence of the strut which blocks the flow between wheels creating a high pressure region. So, the flow passes through the outer shoulder of the wheel with higher velocity creating a low pressure region as depicted in Figure 11(b).

Consider two slices along section A-A and B-B in the XZ plane as shown in Figure 11(c). Section A-A cuts through the strut and the fuselage centerline. While, section B-B is a cut through the center of the port side wheel of the NLG. Figure 12 illustrates the normalized velocity magnitude $\left(U / U_{\infty}\right)$ on section A-A for the three configurations. For Case-2 higher velocity magnitude is observed near the cavity rear wall (compared to magnitude within the cavity); while, for Case-3, low velocity magnitude is formed near the cavity rear wall as the shear layer formed at the cavity leading edge impinges on it. It is observed that the presence of the cavity changes the mean flow features behind the upper strut (near the cavity trailing edge). The flow escapes from the cavity away from the fuselage and interacts with the wake behind the upper strut as seen in Figure 13. For Case-3, due to the fully exposed cavity, the flow behind the upper strut is directed away from the fuselage. However, there are no significant changes in the mean flow features around the components below the steering assembly. Figure 14 shows the trend of velocity magnitude along a line (plane A-A) behind the NLG. A significant difference is observed between Case- 2 and Case3. For the fully exposed cavity, the flow exiting at the cavity trailing edge pushes the high velocity region away from the fuselage. Also, the mean velocity in the upper part of the NLG reduces due to its interaction with the wake from the front door as evident from Figure 15. It is observed that the front doors are at an angle to the incoming flow creating a divergent section which slows down the flow. In addition, the flow separating from the sharp edges of the front doors forms a wake which encloses the rear door and upper components of the NLG. The wake from the front doors further reduces the velocity of the flow before it interacts with other downstream components. This reduces the individual drag induced by the rear doors and the upper components of the NLG, which is shown later (Table 4) in Section IV(C).

As expected, a significant change in the cavity flow features for the partially and fully exposed cavity is observed. For section A-A, zoom-in views of Case-2 and Case-3 cavity mean flow along with streamlines is shown in Figure 16 . For Case-2, as shown in Figure 16(a), two main recirculation zones are formed within the cavity. Generally, for a partially covered cavity, a bigger recirculation region would be observed at the exposed side and another smaller zone near the enclosed front wall. However, the presence of the cavity front partition plays an important role in directing the flow to create the other recirculation zone at approximately half the length of the cavity.

The flow within the fully exposed cavity in Figure 16(b) is quite different to a fundamental open cavity flow as the shear layer does not interact with the cavity trailing edge. The cavity upper wall creates an angle with respect to the free-stream velocity. Also, the leading and trailing edges of the cavity are not in the same horizontal line due to the shape of the fuselage. In addition, a recirculation region is formed downstream of the front partition which delays the interaction of the shear layer with the cavity upper wall. So, if the front partition is absent, there is a possibility that the flow might interact with the cavity upper wall and result in a closed cavity flow. However, in the current scenario, the flow is directed towards the top end of the cavity rear wall resulting in a high surface pressure 
(Figure 16(d)). Although, the cavity partitions are used as a structural support for the NLG, it creates significant changes in the aerodynamics of the fundamental cavity flow.

Figure 16(c) and Figure 16(d) shows the mean pressure contours for Case- 2 and Case-3 respectively. A uniform mean pressure is observed, for the Case-2, in the front part of the cavity. A high pressure region is formed on the cavity rear wall where the flow impinges after interacting with the lower drag brace. Also, a high pressure region at the top corner of the cavity rear wall is noticed as the flow travels towards the cavity upper wall after interaction with the rear wall as confirmed from Figure 16(a). For Case-3, due to the interaction with the shear layer formed at the cavity leading edge, high pressure region is noticed on the upper half of the cavity rear wall. In addition, lower pressure is observed on the front part of the cavity compared to the rear part. An overall higher pressure is observed in the rear part of the cavity for Case-3 when compared with the Case-2. Due to this, the flow exiting at the cavity trailing edge (for Case-3) is further pushed away from the fuselage (compared to Case-2) which is also noticed in Figure 13. Also, a significant increase in total cavity drag coefficient is observed in Case-3 $\left(C_{d}=0.2243\right)$, compared to Case-2 $\left(C_{d}=0.0333\right)$.

Contour plots of Turbulent Kinetic Energy (TKE) on plane A-A are shown in Figure 17. It is seen that, inside the cavity, Case-3 possesses higher TKE compared to Case-2. For all three cases, high TKE is observed between the lower drag stay and the upper strut. Due to this, high TKE is also observed in the wake of the upper strut. Figure 18(b) shows the mean velocity in the $z$ direction on the fuselage center-line plane. It depicts that the flow between the lower drag brace and the upper strut travels towards the fuselage ( $+z$ direction) with a magnitude equivalent to free-stream velocity $(+x$ direction). This flow travelling upwards interacts with the wake of the lower drag stay causing high TKE in the upstream and downstream of the upper strut. Figure 18(c) shows the front view of the NLG with root mean square (RMS) of the pressure coefficient on the upper strut, steering assembly and lower strut. It is observed that, compared to other parts, higher pressure fluctuations (high $C_{p}$ RMS values) are noticed on the upper strut due to the high TKE between the lower drag stay and the upper strut.

In Figure 19, for all cases, it is observed that the flow separation in the wheel wake is asymmetric. A low velocity region is identified behind the upper half of the wheel. Also, the wake from the wheel travels in the upward direction towards the fuselage. From Figure 20(a), it is seen that the torque link lies in close proximity to the upper half of the wheel. The flow structures formed from the wake of the torque link interacts with the upper half of the wheel resulting in the flow asymmetry. In Figure 20(b), high pressure fluctuations are also noticed on the edges of the torque link which are located close to the wheels. In addition, the mean wall shear stress in the $y$ direction on the back face of the wheel is presented in Figure 20(c). It shows that the presence of the torque link does not allow the vortex formation on the upper inner side of the wheel. The wake from the torque link directs the flow (on the upper inner side of the wheel) in the $-y$ direction causing negative wall shear stress.

\section{Aerodynamic loads and unsteady flow}

The mean and the standard deviation (STDEV) of the drag coefficient are presented in Table 4. An increase in the NLG mean drag is observed from Case-1 to Case-2 as more components within the cavity are exposed to the flow. However, a reduction in the mean drag is seen for Case-3. This reduction in the mean drag occurs as the wake of the front doors slows down the flow impinging on the downstream components. However, an increase in load fluctuation is observed as the flow is disrupted by the presence of the front doors. For Case-3, the rear doors and the upper components of NLG are within the wake of the front doors as previously seen in Figure 15. Also, the favorable pressure gradient under the fuselage disappears as the front doors are open. Hence, a reduction is observed in the mean drag forces on NLG for Case-3 (compared to Case-1 and Case-2). The increase in $C_{d}$ STDEV on the NLG for Case-3 is noticed as the wake from the front doors possesses higher unsteadiness compared to the freestream flow. Due to the effect of the change in the pressure gradient under the fuselage, a reduction in wheel $C_{d}$ is observed from Case-1 to Case-3. The relative reduction in the wheel $C_{d}$ from Case- 1 to Case- 2 is $6.6 \%$. For Case-3, the wheel $C_{d}$ is further reduced by $2.9 \%$ (relative to Case-2). The upper strut also shows reduction in the mean drag force with increase in unsteady loads. The steering assembly possesses the most complex structure with sharp edges and is located away from the cavity. Sohankar ${ }^{32}$ proved that the drag coefficient and the shedding frequency of a bluff body with sharp edges are independent of Reynolds number higher than $2 \times 10^{4}$. Hence, both the mean and the standard deviation of the drag coefficient for the steering assembly remain almost similar in all the three cases. Differences in the door loads are observed since they are located in close proximity to the cavity. Also, in Case-3, 
the wake from the front doors interacts with the shear layer from the cavity leading edge. Table 5 shows information regarding the moment on the rear door about its hinge point. Positive moment in all configurations signifies that the door is pushed outwards which is helpful in landing gear freefall scenarios. A significant increase in the mean and the STDEV of the door moment is also observed for Case-3 compared to other cases.

Table 4: Drag coefficient on NLG and individual parts, mean and standard deviation.

\begin{tabular}{|l|c|c|c|c|c|c|}
\hline \multicolumn{1}{|c|}{ COMPONENT } & \multicolumn{2}{|c|}{ Case-1 (sealed) } & \multicolumn{2}{c|}{$\begin{array}{c}\text { Case-2 (partially } \\
\text { exposed) }\end{array}$} & \multicolumn{2}{c|}{ Case-3 (fully exposed) } \\
\hline & $\mathbf{C}_{\mathbf{d}}$ mean & $\mathbf{C}_{\mathbf{d}}$ STDEV & $\mathbf{C}_{\mathbf{d}}$ mean & $\mathbf{C}_{\mathbf{d}}$ STDEV & $\mathbf{C}_{\mathbf{d}}$ mean & $\mathbf{C}_{\mathbf{d}}$ STDEV \\
\hline NLG total & 1.1209 & 0.0242 & 1.1378 & 0.0230 & 1.0666 & 0.0341 \\
\hline Front door LHS & - & - & - & - & 0.1034 & 0.0059 \\
\hline Rear door LHS & 0.0788 & 0.0029 & 0.0698 & 0.0036 & 0.0512 & 0.0082 \\
\hline Upper strut & 0.1510 & 0.0098 & 0.1434 & 0.0081 & 0.1244 & 0.0136 \\
\hline Lower strut & 0.1951 & 0.0021 & 0.1942 & 0.0024 & 0.1911 & 0.0028 \\
\hline Side stay LHS & 0.0640 & 0.0033 & 0.0835 & 0.0065 & 0.0602 & 0.0127 \\
\hline Steering assembly & 0.1466 & 0.0032 & 0.1472 & 0.0032 & 0.1460 & 0.0031 \\
\hline Torque link & 0.0011 & 0.0024 & 0.0054 & 0.0061 & 0.0053 & 0.0048 \\
\hline Upper drag stay & - & - & 0.0207 & 0.0010 & 0.0423 & 0.0147 \\
\hline Lower drag stay & 0.0530 & 0.0022 & 0.0526 & 0.0021 & 0.0490 & 0.0052 \\
\hline Wheel LHS & 0.2178 & 0.0110 & 0.2035 & 0.0106 & 0.1975 & 0.0094 \\
\hline
\end{tabular}

Table 5: Rear door moment with respect to the door hinge.

\begin{tabular}{|l|c|c|c|c|c|c|}
\hline \multirow{2}{*}{ COMPONENT } & \multicolumn{2}{|c|}{ Case-1 (sealed) } & \multicolumn{2}{c|}{$\begin{array}{c}\text { Case-2 (partially } \\
\text { exposed) }\end{array}$} & \multicolumn{2}{c|}{ Case-3 (fully exposed) } \\
\hline & $\mathbf{C}_{\mathbf{m}}$ mean & $\mathbf{C}_{\mathbf{m}}$ STDEV & $\mathbf{C}_{\mathbf{m}}$ mean & $\mathbf{C}_{\mathbf{m}}$ STDEV & $\mathbf{C}_{\mathbf{m}}$ mean & $\mathbf{C}_{\mathbf{m}}$ STDEV \\
\hline Rear door LHS & 0.1294 & 0.0049 & 0.1198 & 0.0082 & 0.1844 & 0.0296 \\
\hline
\end{tabular}

As the interaction of the cavity and the NLG occurs close to the upper strut, it changes the dominant shedding frequency immediately downstream which affects the nature of the unsteady loads acting on it. Figure 21 represents the PSD (power spectrum density) plot for a pressure probe behind the upper strut. The plot is produced by averaging over 7 sample windows with $50 \%$ overlap acquiring a frequency resolution of $100 \mathrm{~Hz}$. No sharp peak is identified in any of the configurations, however broad peaks are observed which spread over a wide range of frequencies. In Figure 21, an increase in the overall fluctuation is observed from Case-1 to Case-3. This increase in the PSD level (behind the upper strut) is due to the presence of a partially exposed and a fully exposed cavity in Case-2 and Case-3 respectively. Figure 22 shows a PSD plot (Case-3) comparing two symmetric points (W7 and W11) on the upper and lower half of the wheel surface. The probe on the upper half of the wheel (W7) possesses higher pressure fluctuations compared to the symmetrically located probe on the lower half. The high PSD levels on W7 can be associated with the wake of from torque link impinging on the wheel. The interaction of the torque link wake with the inner side of the wheels (in the downstream) can be noticed in Figure 23 which shows the vorticity ( $z$ direction) contours on a plane $\mathrm{Z}=-0.445$. This interaction triggers an early flow separation on the upper half of the wheel (compared to the lower half) resulting in an asymmetric flow.

Figure 24 shows PSD plots for the probe located at the cavity trailing edge. The theoretical frequency at various modes obtained from Rossiter's formula is also shown in the plots. The length $(L)$ of the cavity is taken as the shortest distance between the cavity leading and trailing edges. It is inferred from Figure 24(a) that frequency obtained from different Rossiter modes match closely to the small fluctuation peaks obtained from the simulation. However, peaks at mode 5 and 6 do not match well. This disagreement may be caused due to the presence of components like upper strut, upper drag stay and lower drag stay. Based on the assumed St of 0.125-0.15 for components with sharp edges and 0.2 for cylindrical shaped parts, these components induce shedding frequency 
ranging between 1000-1600 Hz. The low frequency Rossiter mode $(n=1)$ does not agree well as the PSD plot is averaged over several windows which reduce the signal noise, but results in poor resolution at lower frequency. In summary, Case-2 represents an open cavity flow with pressure fluctuation peaks in close agreement with Rossiter's formula. On the contrary, in Figure 24(b), pressure fluctuation peaks are not present at the predicted resonance frequency from Rossiter's formula. This is expected due to the absence of interaction between the shear layer and the cavity trailing edge in the fully exposed cavity configuration (Case-3). The fluctuations are spread over a broad range of frequencies due to the complex interaction between the shear layer, the front door wake and the cavity partition. Two peaks are observed at $1200 \mathrm{~Hz}$ and $1600 \mathrm{~Hz}$ respectively. These frequencies are probably associated with the wake from the upper strut and the drag stay. The origin of these PSD peaks can be confirmed from Figure 25 (PSD plot on a probe behind upper strut) as similar pressure fluctuation peaks in the high frequency range are observed downstream of the dray stay and the upper strut.

\section{Conclusion}

DDES simulations on a complex NLG model were performed with three different geometry configurations - (1) a fully extended NLG with front doors closed and cavity sealed, (2) a fully extended NLG with front doors closed and cavity partially exposed and (3) a fully extended NLG with front doors open and cavity fully exposed. The simulation results match closely to the experimental data with $2 \sim 3 \%$ of relative error for the drag and moment coefficients. The interaction between the torque link and the wheel causes asymmetry in the flow behind the wheel. It is observed that the cavity structural partition plays an important role in changing the mean flow features within the cavity. An open cavity flow is observed for the partially exposed cavity with oscillation frequencies agreeing well with the Rossiter modes. For the fully exposed cavity, the shear layer from the cavity leading edge does not interact with the trailing edge due to the slant angle on the cavity upper wall; hence, an open cavity flow behavior is not observed. Compared to the sealed cavity case, the unsteadiness in the flow increases for the partially exposed cavity (unsteady loads increases further when it is fully exposed). With change in the cavity configuration, noticeable changes in the steady and unsteady loads were observed on the rear doors, drag braces and the upper strut. Small changes in the drag forces are observed on other individual components except the steering assembly. This work currently focuses on the investigation of the cavity flow on a fully extended landing gear. In future, this study can be further widened by considering the landing gear at various angles during its retraction and extension phases. In addition, the effect of the cavity partition and the upper wall slant angle can be further explored in a fundamental manner using a simplified cavity model.

\section{Acknowledgments}

This study is performed as a part of the ALGAAP project jointly funded by Airbus and TSB UK (now known as Innovate UK). The authors acknowledge the cooperation from Airbus UK for providing the geometry and the wind tunnel data. Special thanks to Mr. Alan Shepherd and Mr. Yasir Arfeen from Airbus UK for their support and guidance during this study. The authors acknowledge the use of the IRIDIS High Performance Computing Facility, and associated support services at the University of Southampton.

\section{References}

${ }^{1}$ Rossiter, J., "Wind tunnel experiments on the flow over rectangular cavities at subsonic and transonic speeds.," Royal Aircraft Establishment, TR 64037, 1964.

${ }^{2}$ Green, J. E., "Civil aviation and the environmental challenge," Aeronautical Journal, Vol. 107, No. 1072, 2003.

3 Dobrzynski, W., "Almost 40 Years of Airframe Noise Research:What Did We Achieve?," Journal of Aircraft, Vol. 47, No. 2, 2010, pp. 353-367.

${ }^{4}$ Block, P., J., W., "Noise responce of cavities of varying dimensions at subsonic speeds," Technical Note D-8351, 1976.

${ }^{5}$ Ahuja K., K., and Mendoza, J., "Effects of cavity dimensions, boundary layer and temperature on cavity noise with emphasis on benchmark data to validate computational aeroacoustics," NASA, CR 4653, 1995. 
${ }^{6}$ Heller, H., H., Holmes G., D., and Covert, E., E., "Flow induced presure oscillations in shallow cavities," Air Force Flight Dynamics Laboratory, TR AFFDL-TR-70-104, 1970.

${ }^{7}$ Tam C., and Block, P., "On the tones and pressure oscillations induced by flow over rectangular cavities," Journal of Fluid Mechanics, Vol. 89, No. 2, 1978, pp. 373-399.

${ }^{8}$ Block, P., "Noise response of cavities of varying dimensions at subsonic speeds," TN D-8351, NASA, 1976.

${ }^{9}$ Block, P., "Measurements of the tonal component of cavity noise and comparison with theory," TP 1013, NASA, 1977.

${ }^{10}$ Sarohia, V., "Experimental investigation of oscillations in flow over shallow cavities," AIAA Journal, Vol. 15, No. 7, 1977, pp. 984-991.

${ }^{11}$ Rubio, G., Roeck W., De., Baelmans M., and Desmet W., "Numerical identification of flow-induced oscillation modes in rectangular cavities using large eddy simulation," International Journal for Numerical Methods in Fluids, Vol. 53, 2007, pp. 851-866.

${ }^{12}$ Rowley, C., Colonius, T. and Basu, A., "On self-sustained oscillations in two-dimensional compressible flow over rectangular cavities," Journal of Fluid Mechanics, Vol. 455, 2002, pp. 315-346.

${ }^{13}$ Gharib, M., and Roshko, A., "The effect of flow oscillations on cavity drag," Journal of Fluid Mechanics, Vol. 177, 1987, pp. 501-530.

${ }^{14}$ Langtry, R., and Spalart, P., "Detached eddy simulation of a nose landing gear cavity," IUTAM bookseries: proceedings of the IUTAM Symposium "Unsteady Separated Flows and their Control," Corfu, Greece, 18-22 June 2007 Dordrecht;New York; Springer, 2009, pp. 357-366.

${ }^{15}$ Lawson S., J., and Barakos, G., N., "Review of numerical simulations for high-speed, turbulent cavity flows," Progress in Aerospace Sciences, Vol. 27, No. 3, 2011, pp. 186-216.

${ }^{16}$ Imamur, T., Hirai, T., Amemiya, K., Yokokawa, Y., Enomoto S., and Yamamoto, K., "Aerodynamic and aeroacoustic simulations of a two-wheel landing gear," Procedia Engineering, Vol. 6, No. 0, 2010, pp. 293-302.

${ }^{17}$ Murayama, M., Yokokawa, Y., Kato, H,. Ura, H., Uchida, H., Yamamoto, K., Abe, K., and Wu, L., "Computational and Experimental Study on Noise Generation from Tire-Axle Regions of a Two-wheel Main Landing Gear," in 17th AIAA/CEAS Aeroacoustics Conference (32nd AIAA Aeroacoustics Conference) 2011.

${ }^{18}$ Fortin, F., Syms, J., Clark, C. and McIlwain S., "CFD investigation of noise around landing gear," in 18th AIAA/CEAS Aeroacoustics Conference (33rd AIAA Aeroacoustic Conference) 2012.

${ }^{19}$ Tabesh, M. and Waller, G., "Numerical Investigation of 1/3- and Full-Scale Partially-Dressed Small Business Jet Landing Gears," in 18th AIAA/CEAS Aeroacoustics Conference (33rd AIAA Aeroacoustic Conference) 2012.

${ }^{20}$ Thomas Van de, V., John, L., Dean, P., and Fred M., "Computational aeroacoustic analysis of a 1/4 scale G550 nose landing gear and comparison to NASA and UFL wind tunnel data," in 15th AIAA/CEAS Aeroacoustics Conference Miami, Florida: 2009.

${ }^{21}$ Vuillot, F., Houssen, F., Manoha, E., Redonnet, S., and Jacob, J., "Application of the CEDRE unstructured flow solver to landing gear unsteady flow and noise prediction," in 17th AIAA/CEAS Aeroacoustics Conference (32nd AIAA Aeroacoustic Conference) 2011.

${ }^{22}$ Vuillot, F., Lupogalzoff, N., Luquet, D., Sanders, L., Manoha, E., and Redonnet, S., "Hybrid CAA solutions for nose landing gear noise," in 18th AIAA/CEAS Aeroacoustics Conference (33rd AIAA Aeroacoustic Conference) 2012. 
${ }^{23}$ Vatsa, V., Lockard, D., and Khorrami, R., "Application of FUN3D solver for aeroacoustic simulation of a nose landing gear configuration," in 17th AIAA/CEAS Aeroacoustics Conference (32nd AIAA Aeroacoustic Conference) 2011.

${ }^{24}$ Houghton, T., and UnderHill, R., "Nose landing gear computational and experimental results comparison", Report by Frazer-Nash Consultancy submitted to Airbus UK, FNC 39895/37924R, 2011

${ }^{25}$ Liu, W., Van Mierlo, K., Zhang, X., "Nose landing gear CFD study", Internal report by ANTC (university of Southampton) submitted to Airbus UK, 2012

${ }^{26}$ Chow, P., Cross, M., and Pericleous K., "A natural extension of the conventional finite volume method into polygonal unstructured meshes for CFD application," Applied Mathematical Modelling, Vol. 20, No. 2, 1996, pp. 170-183.

${ }^{27}$ Spiegel, M., Redel, T., Zhang, Y., J., Struffert, T., Hornegger, J., Grossman, R., G., Doerfler, A., and Karmonik, C., "Tetrahedral vs. polyhedral mesh size evaluation on flow velocity and wall shear stress for cerebral hemodynamic simulation," Computer methods in Biomechanics and Biomedical Engineering, Vol. 14, No. 1, 2011, pp. 9-22.

${ }^{28}$ Winkler, C., Dorgan, A., Mani, M., Larssen, J. and Langtry, R., " Grid sensitivity of the rudimentary landing gear using unstructured finite volume methods," in 18th AIAA/CEAS Aeroacoustics Conference (33rd AIAA Aeroacoustic Conference) 2012.

${ }^{29}$ OpenCFD Ltd. Standard solvers. URL http://www.openfoam.org/features/standard-solvers.php.

${ }^{30}$ Spagnolo, S., Zhang, X., Hu, Z. and Angland, D., " Numerical simulations of single and tandem wheels for aerodynamic load predictions," in 21st AIAA/CEAS Aeroacoustic Conference, 2015

${ }^{31}$ Bradfield, M., "Nose landing gear R\&T wind tunnel test RLG80NLG_1-01,"Airbus report RP1027795, 2010.

32 Sohankar,A. "Flow over a bluff body from moderate to high Reynolds numbers using large eddy simulation,"Computers \& Fluids, Vol. 35, No. 10, 2006 pp. 1154-1168. 


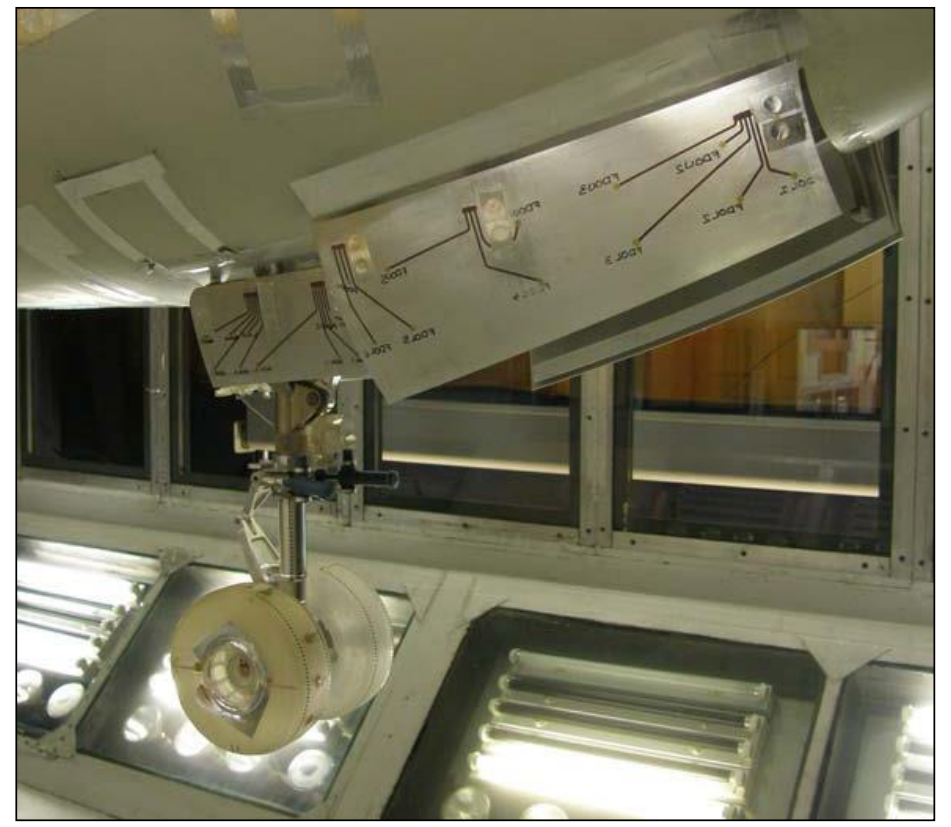

Figure 1: NLG wind tunnel model.
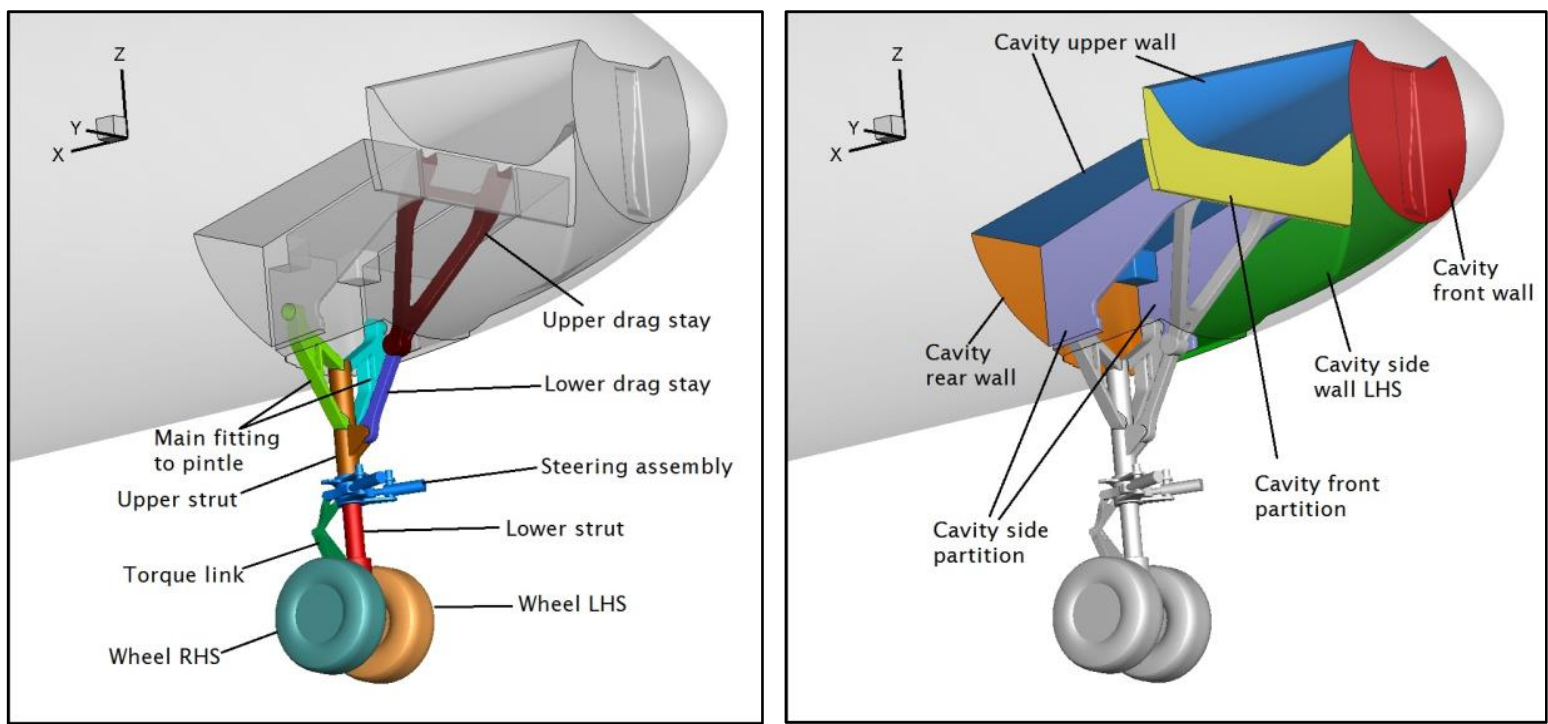

Figure 2: CFD configuration with NLG and cavity parts. 

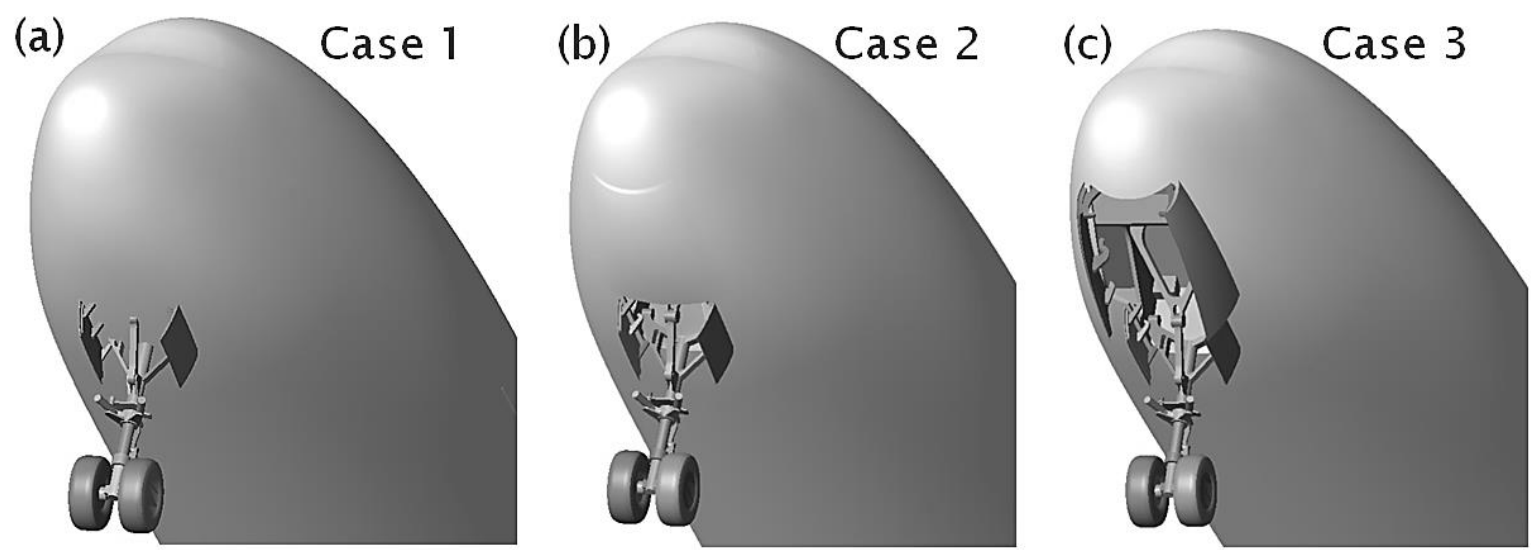

Figure 3: (a) NLG with cavity sealed, (b) NLG with front doors closed and cavity partially exposed and (c) NLG with front doors open and cavity fully exposed.
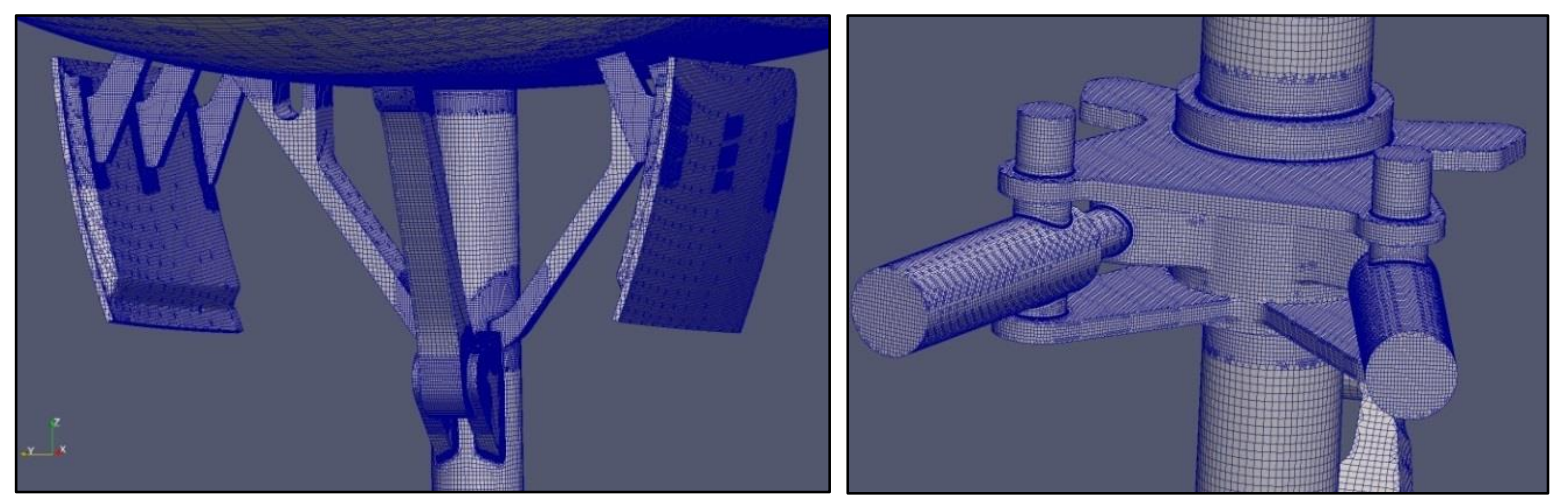

Figure 4: NLG surface mesh on upper strut, rear door and steering assembly.
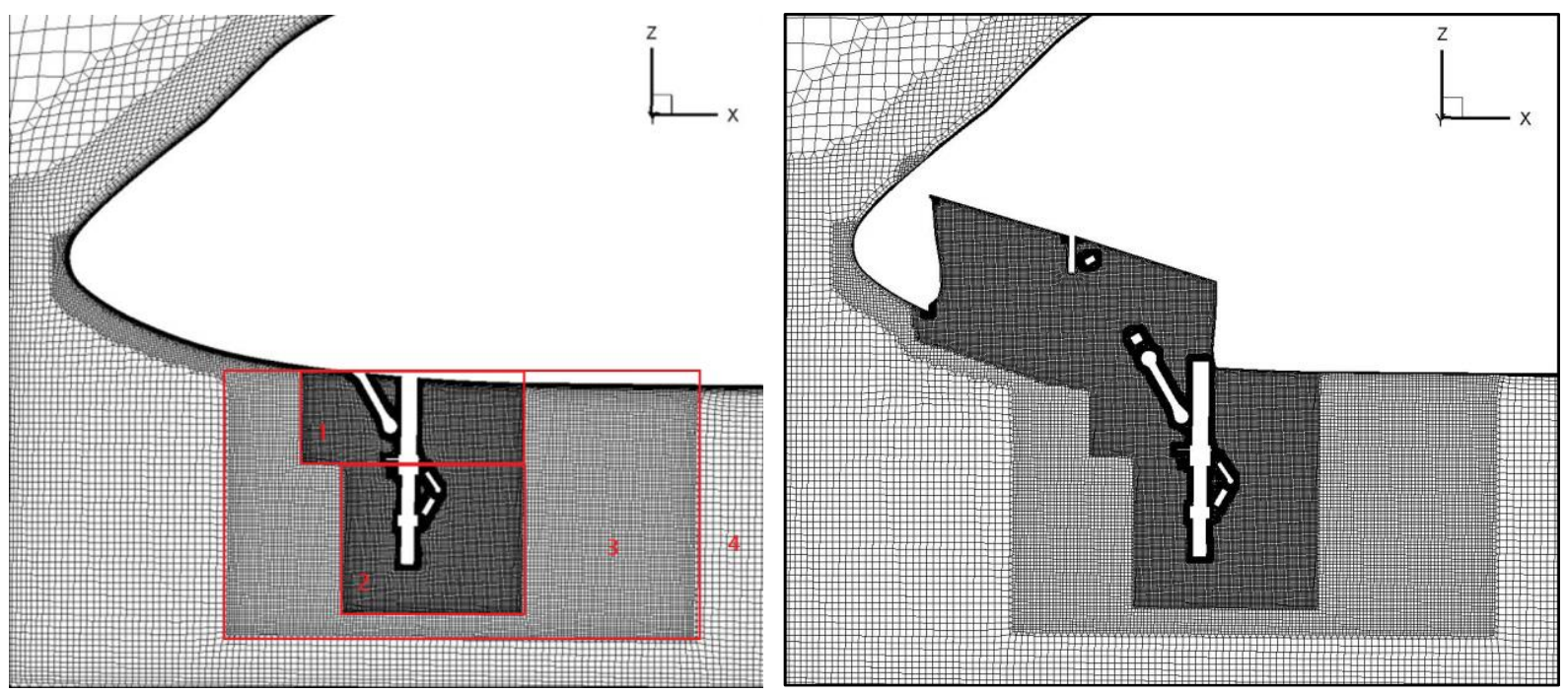

Figure 5: NLG volume mesh cut at fuselage center. 


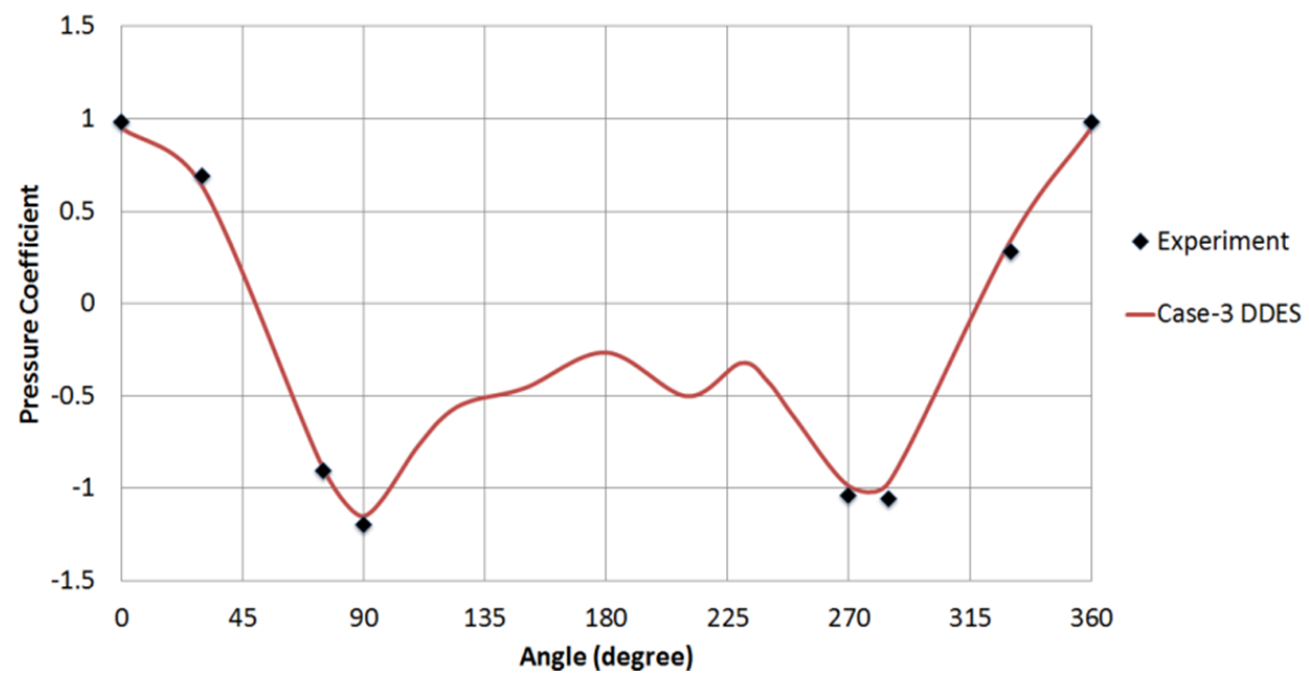

Figure 6: Pressure coefficient on the wheel center-line.
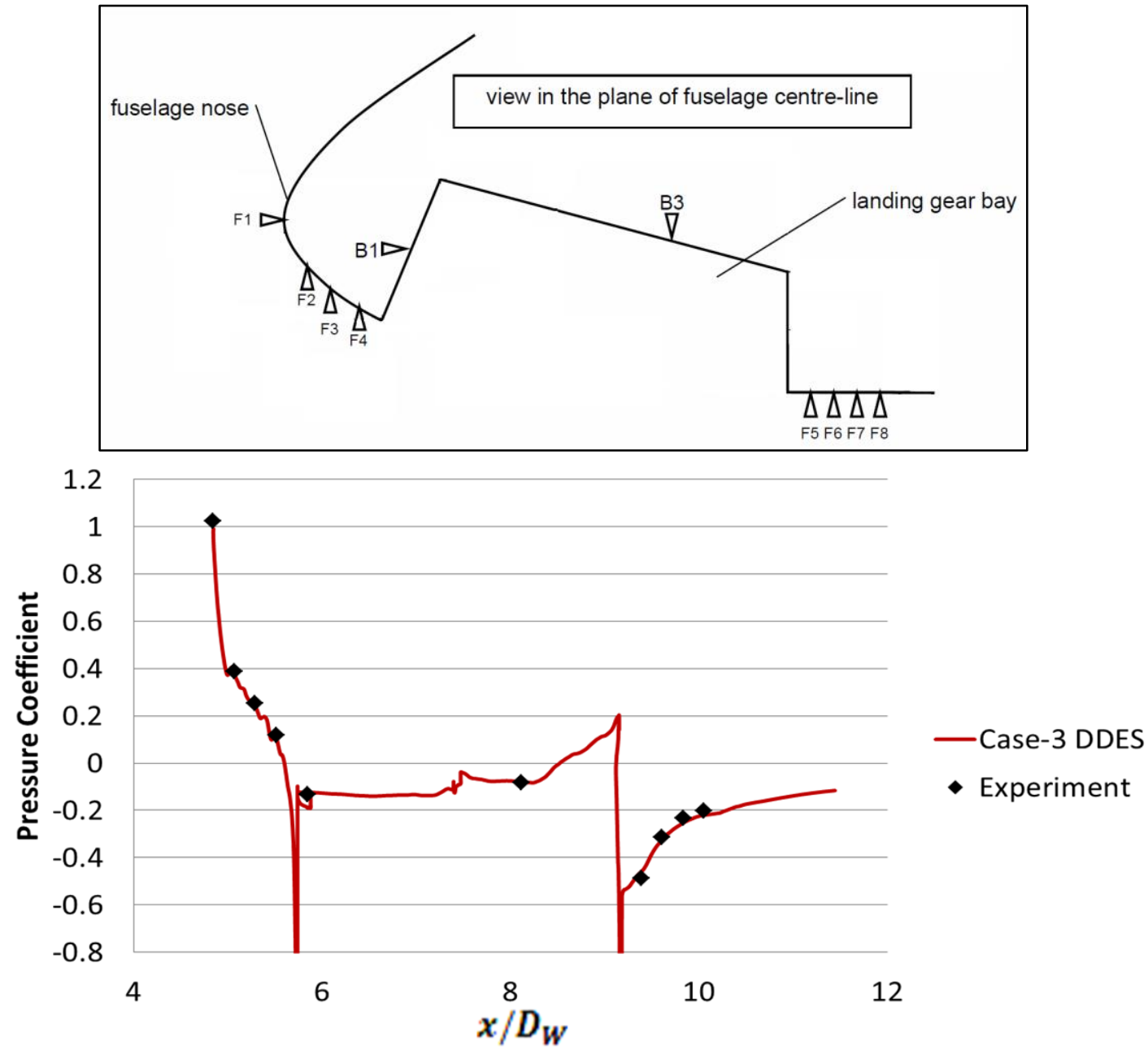

Figure 7: Pressure coefficient on the fuselage and the cavity pressure probes. 

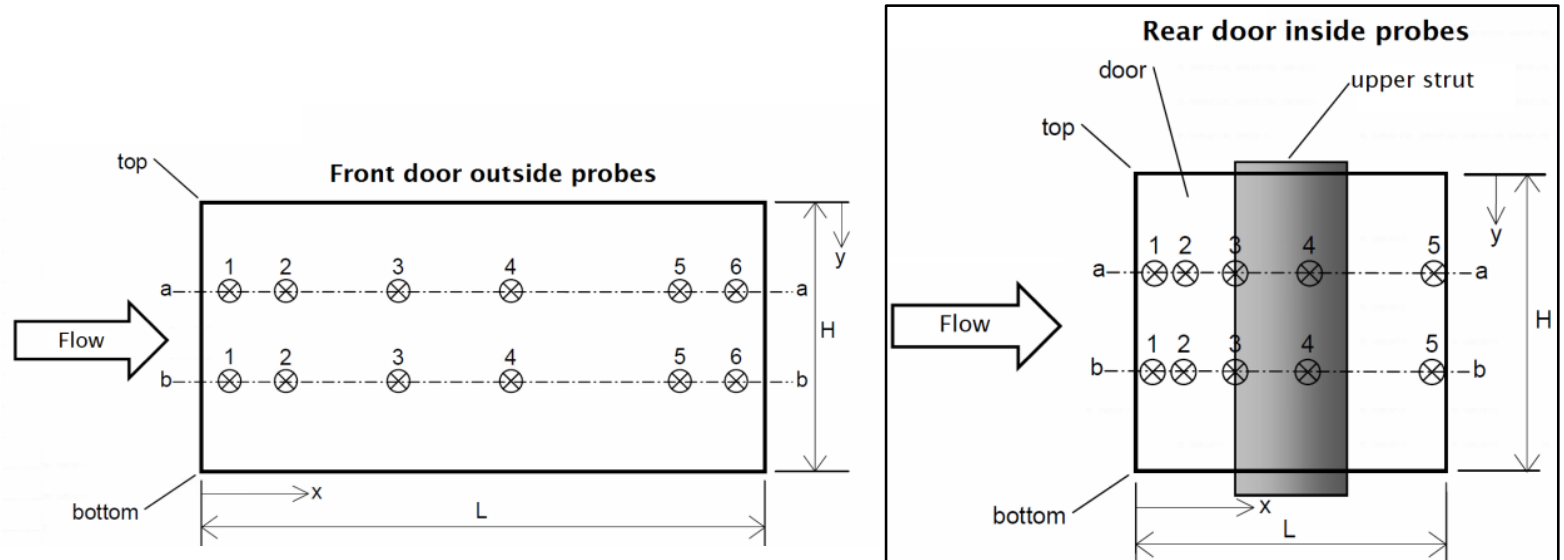

Figure 8: Front door and rear door probe locations (upper and lower rows).
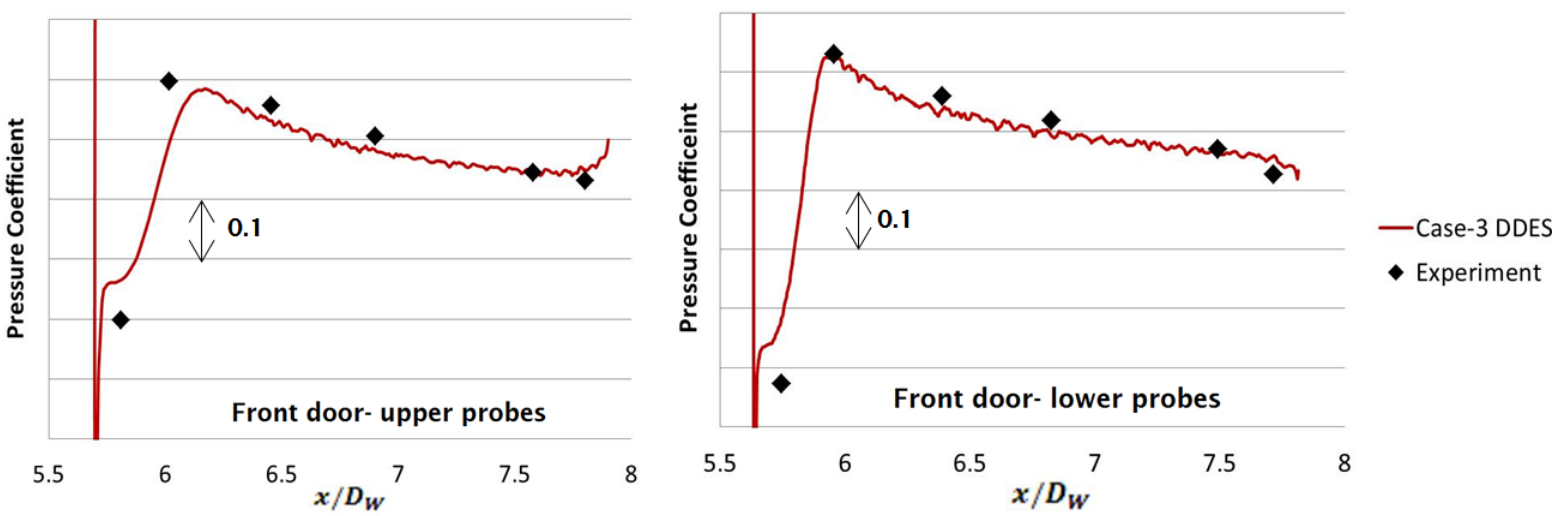

Figure 9: Pressure coefficient for the upper and the lower rows of the front door probes
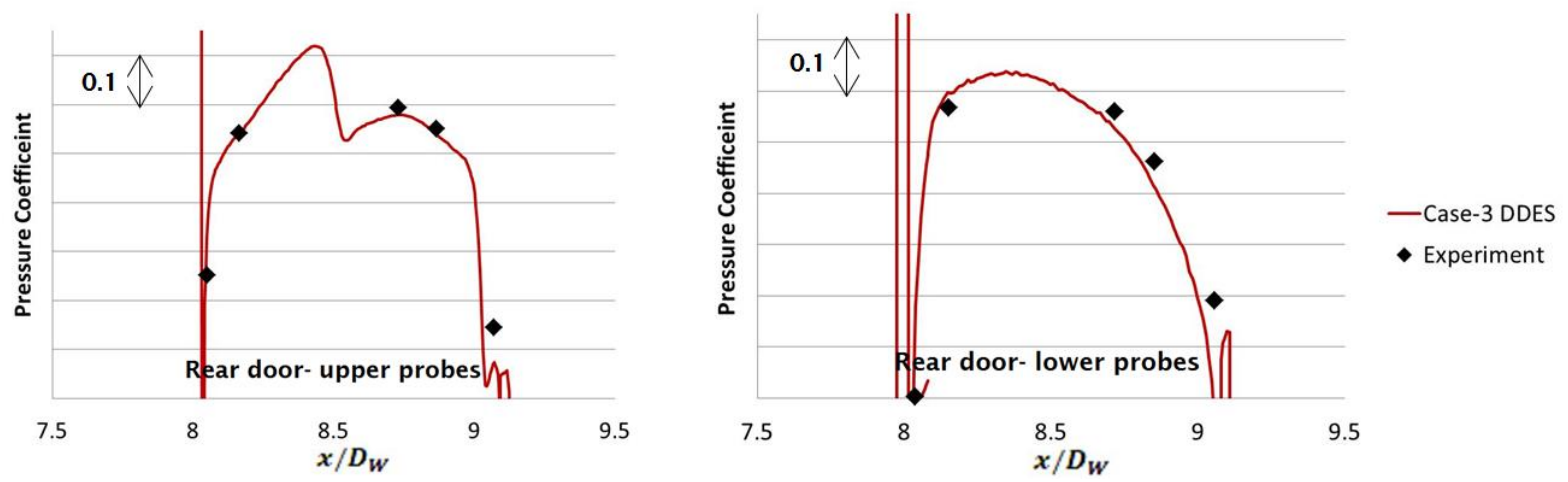

Figure 10: Pressure coefficient for the upper and the lower rows of the rear door probes. 

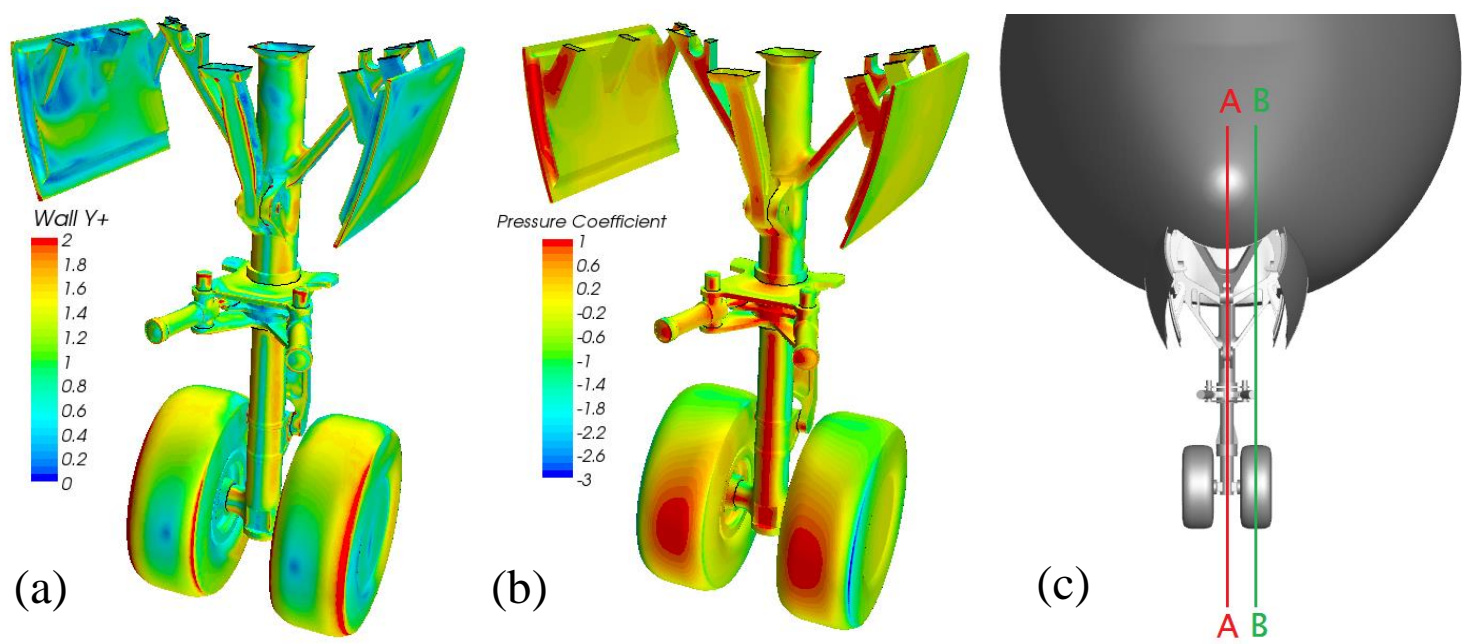

Figure 11: (a) Wall y+ contours, (b) pressure coefficient contours and (c) Location of plane A-A and B-B.
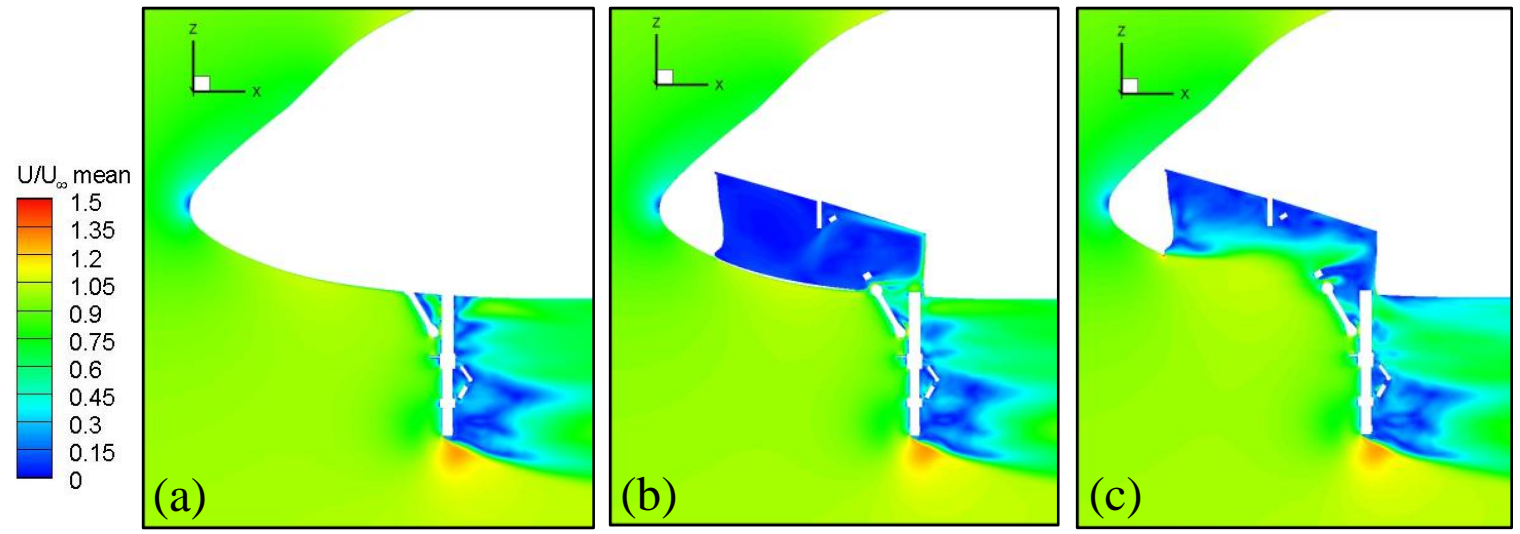

Figure 12: Mean velocity magnitude contours on slice A-A, (a) Case-1, (b) Case-2 and (c) Case-3.
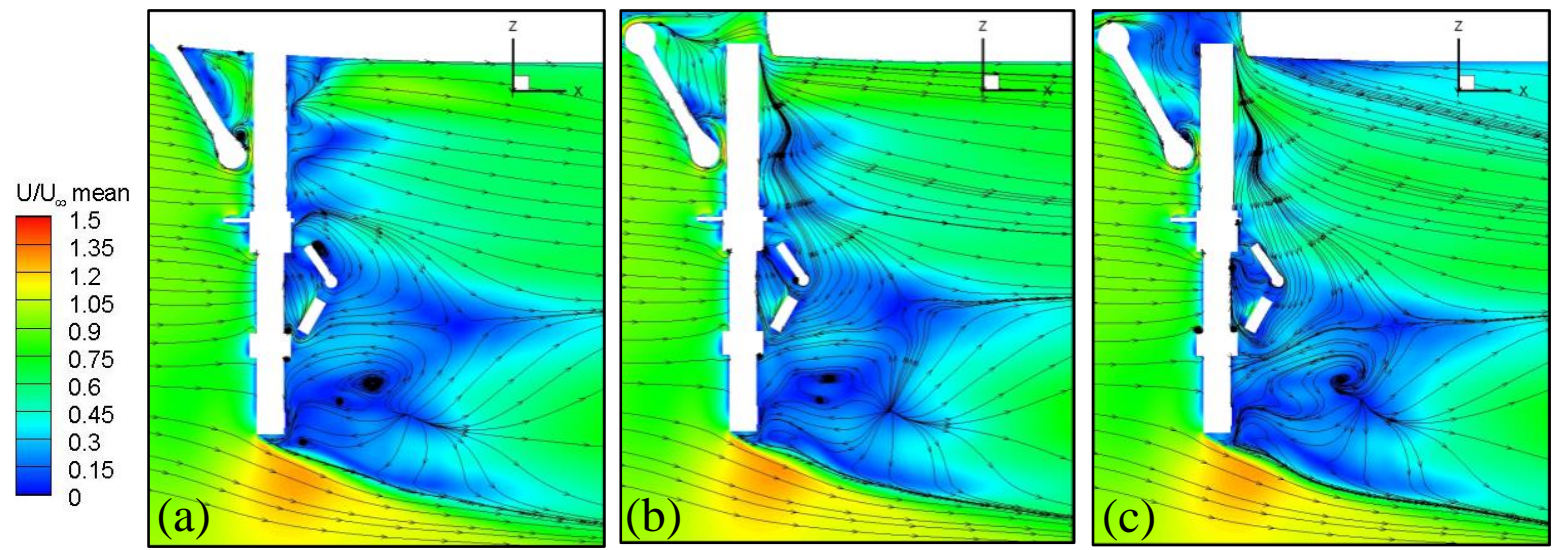

Figure 13: Mean velocity magnitude contours with streamlines on slice A-A, (a) Case-1, (b) Case-2 and (c) Case-3. 


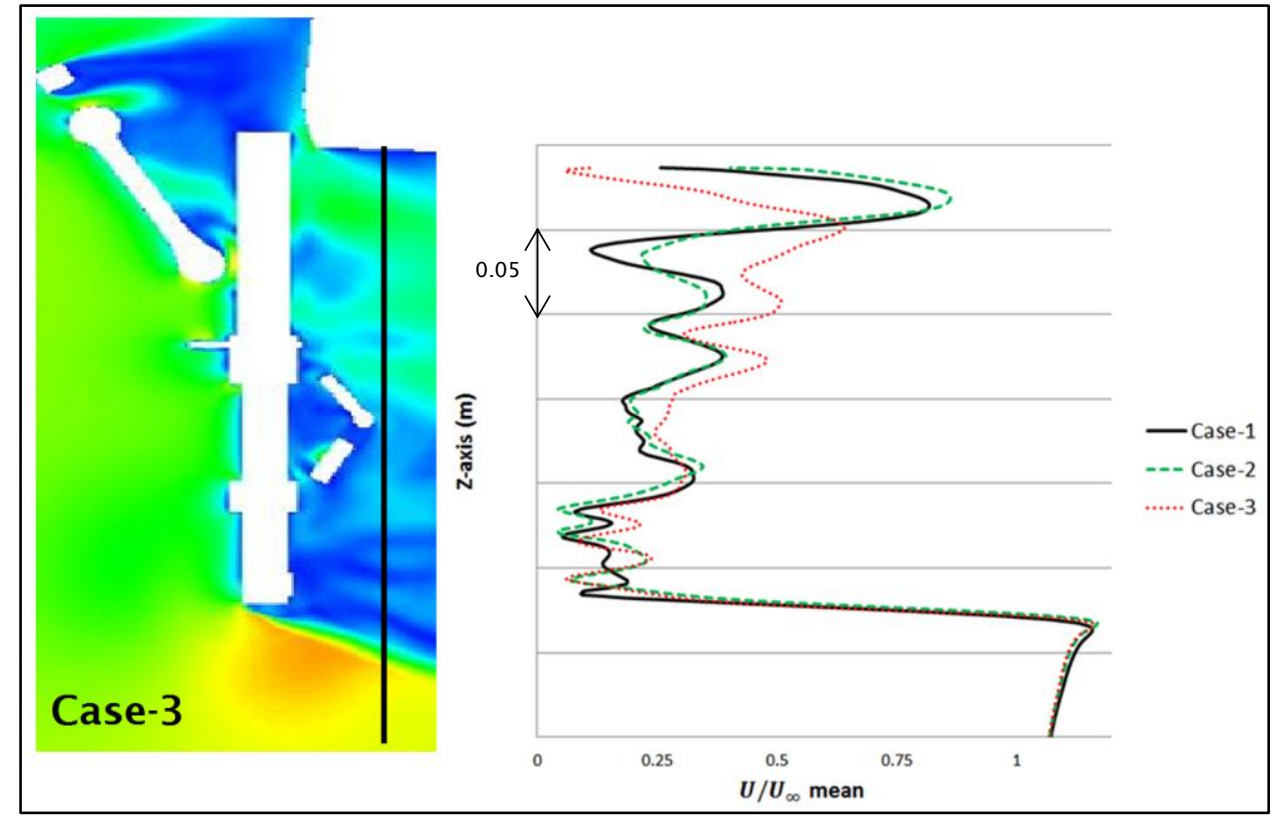

Figure 14: Mean velocity profiles behind the NLG along a line on plane A-A.
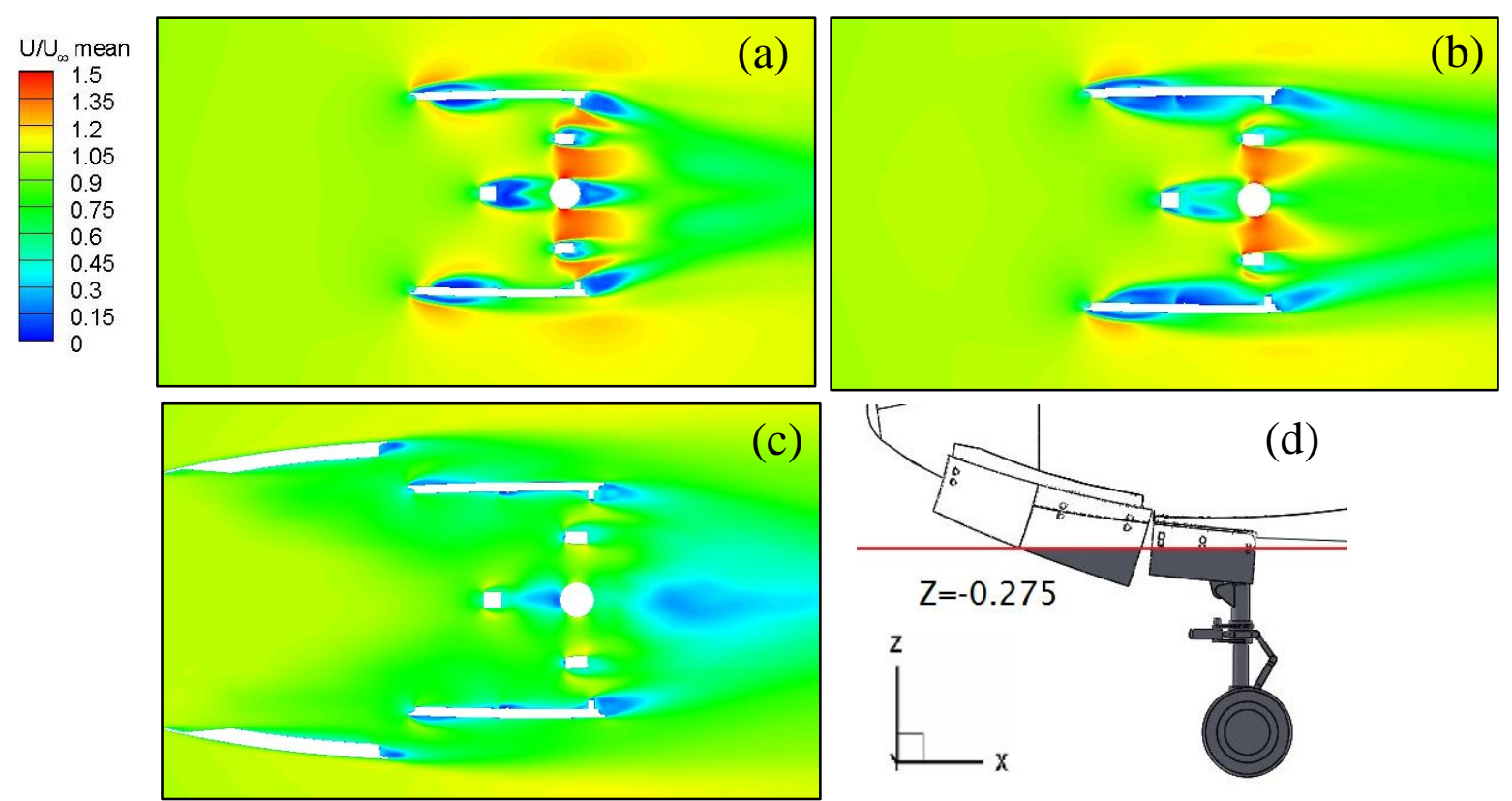

Figure 15: Plane $Z=-0.275$ mean velocity magnitude- (a) Case-1, (b) Case-2, (c) Case-3 and (d) plane location. 

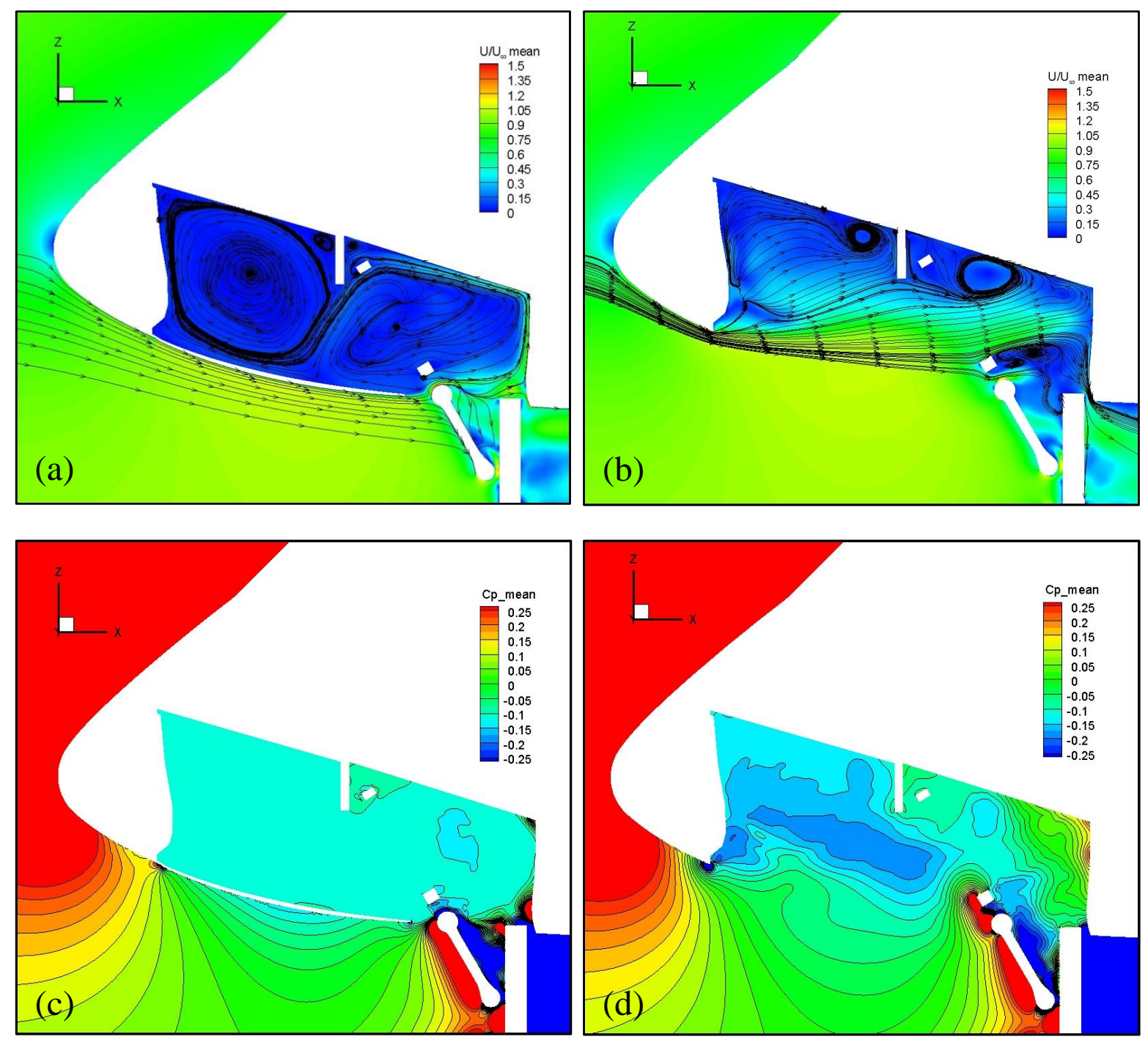

Figure 16: Plane A-A, streamlines with velocity magnitude contours (a) Case-2 and (b) Case-3; mean pressure coefficient contours (c) Case-2 (d) Case-3

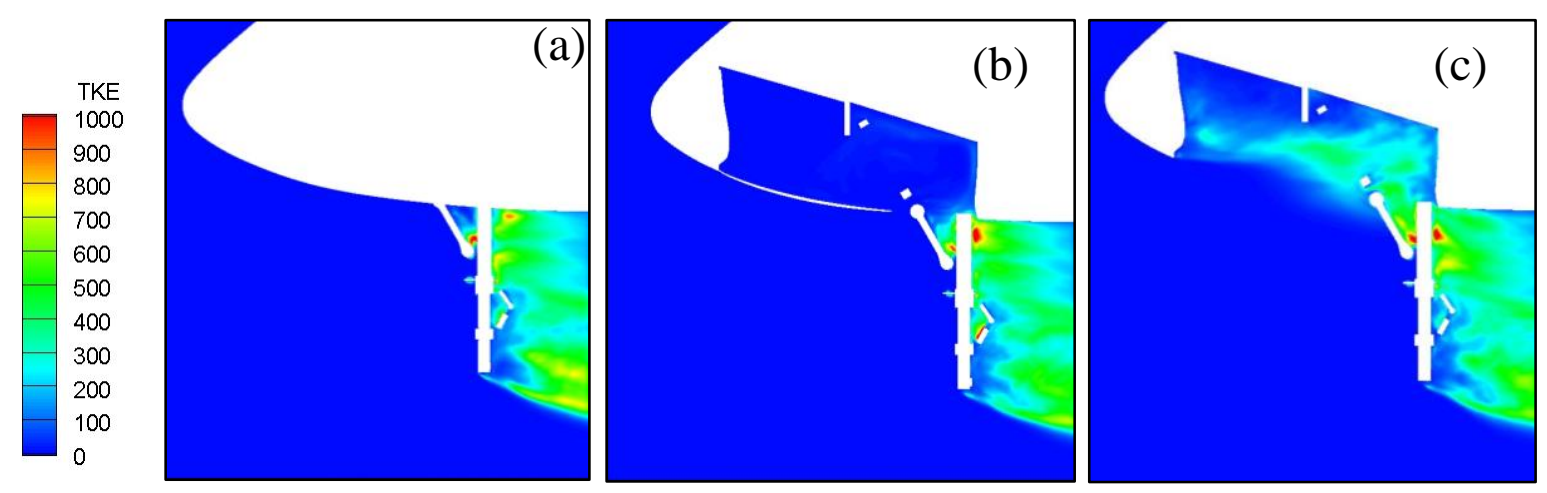

Figure 17: Turbulent kinetic energy on plane A-A, (a) Case-1, (b) Case-2 and (c) Case-3. 
(a)

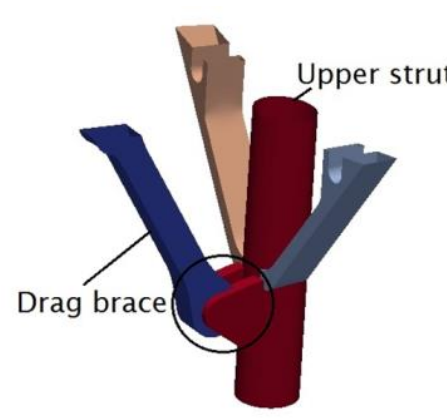

(b)

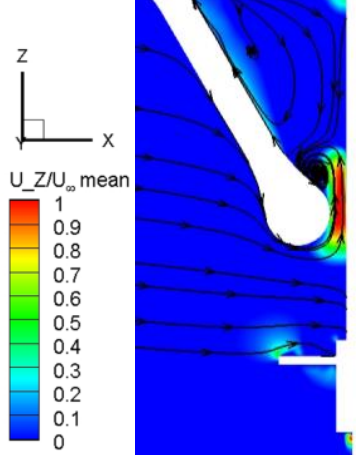

(c)

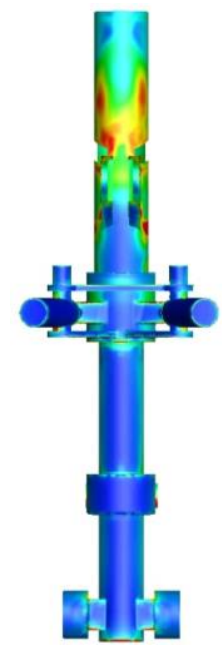

Figure 18: (a) Position of lower drag brace and upper strut, (b) Mean velocity magnitude in $Z$ direction with streamlines, (c) RMS of pressure coefficient contours on upper strut, steering assembly and lower strut.

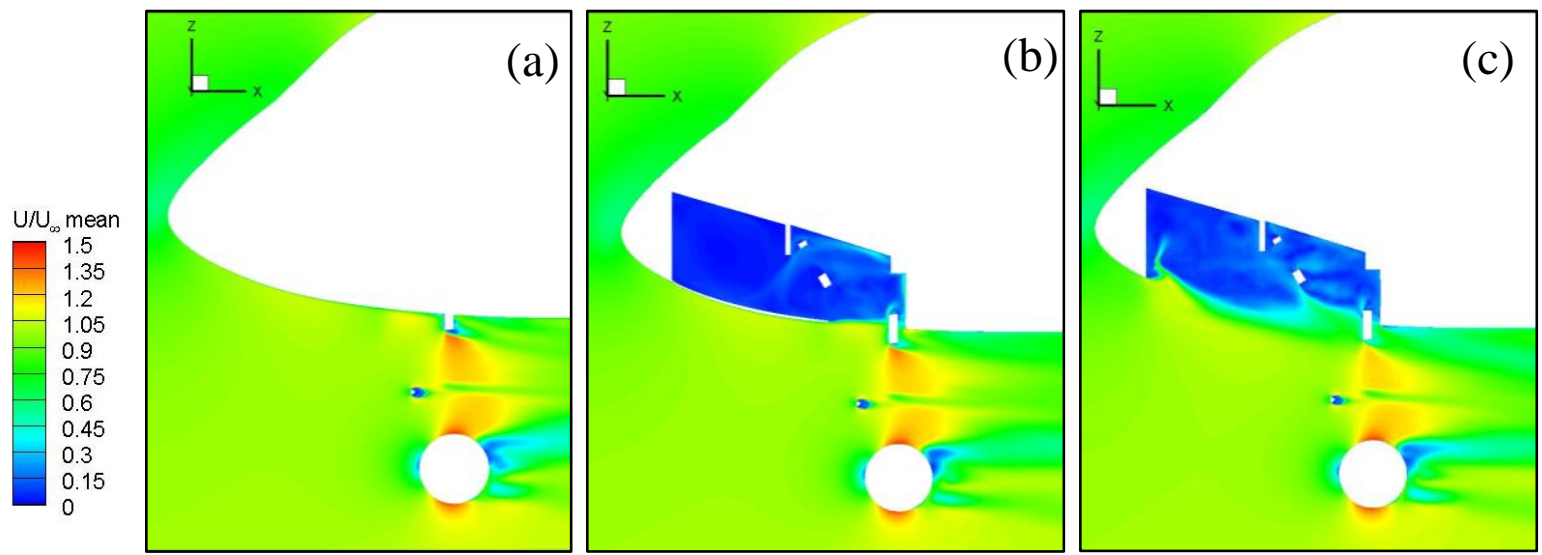

Figure 19: Mean velocity magnitude contours on $\mathrm{XZ}$ plane slice B-B, (a) Case-1, (b) Case-2 and (c) Case-3.
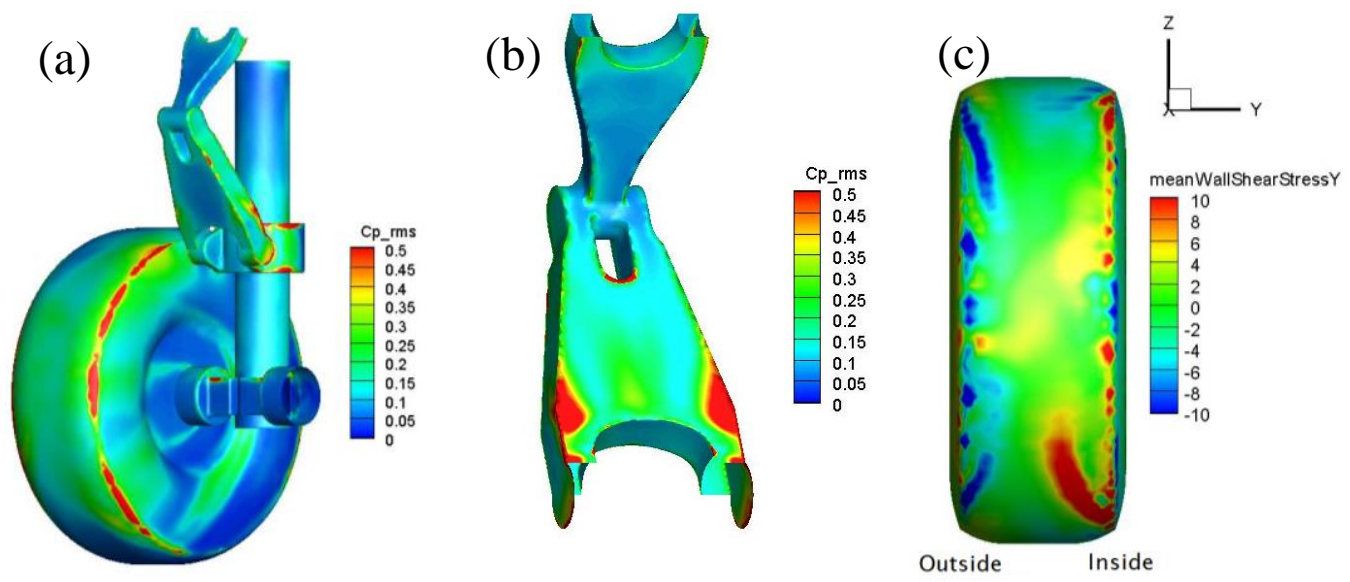

Figure 20: (a) RMS of pressure coefficient on back of wheel, lower strut and torque link, (b) RMS of pressure coefficient on front side of the torque link, (c) Mean wall shear stress in $y$ direction on back of the wheel. 


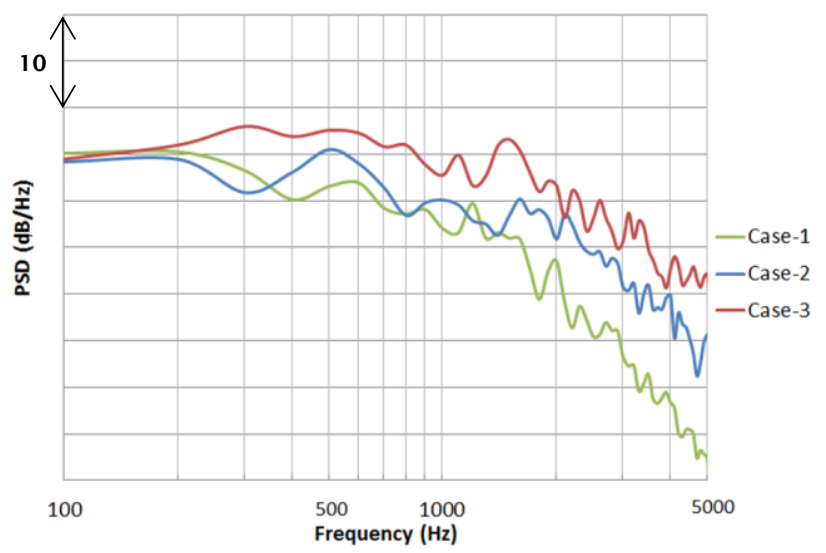

Figure 21: Power spectral density of pressure on the upper strut surface.

(a)
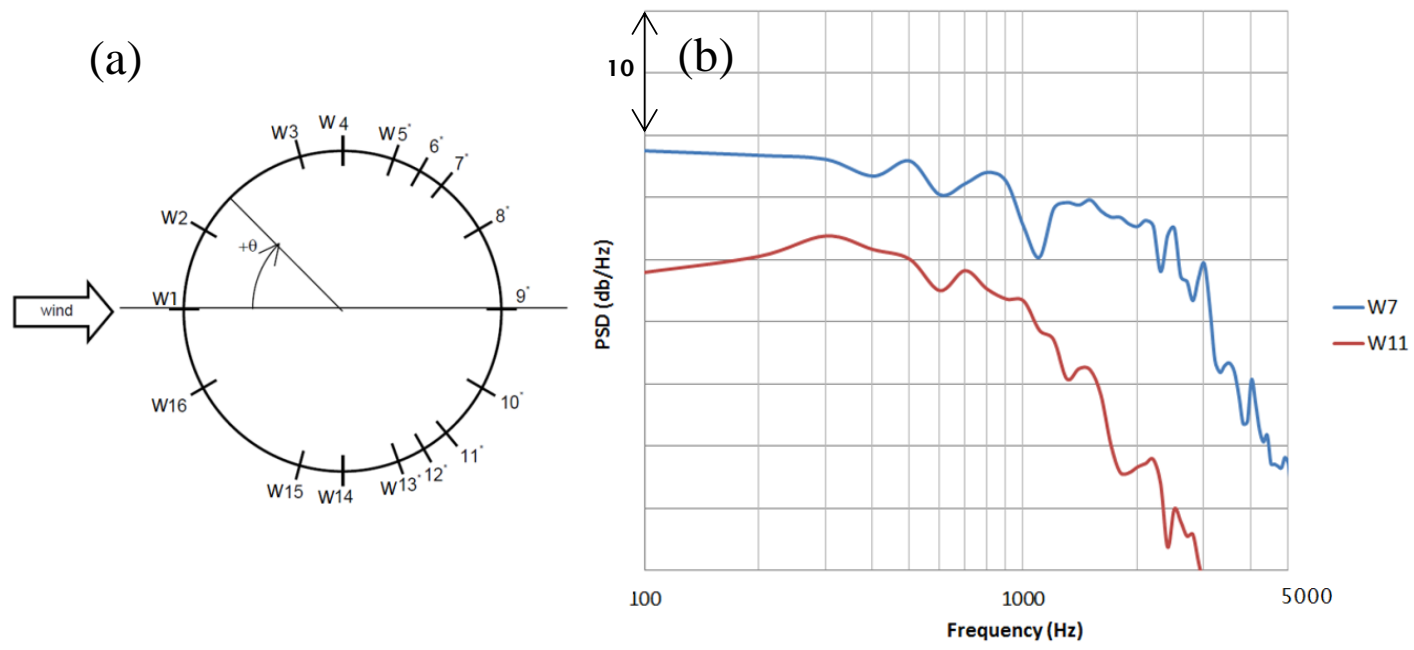

Figure 22: PSD plot (pressure) on wheel surface, probe W7 and W11 (Case-3).

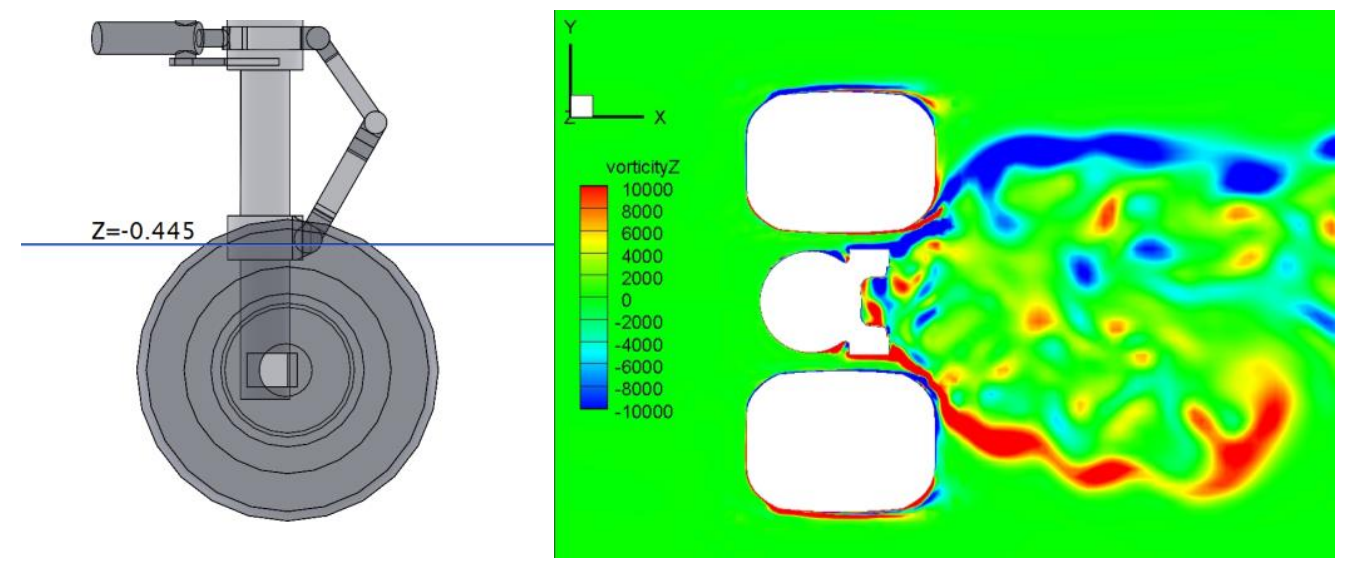

Figure 23: Voritcity ( $z$ direction) contour plot on plane $Z=-0.455$ (Case-3). 

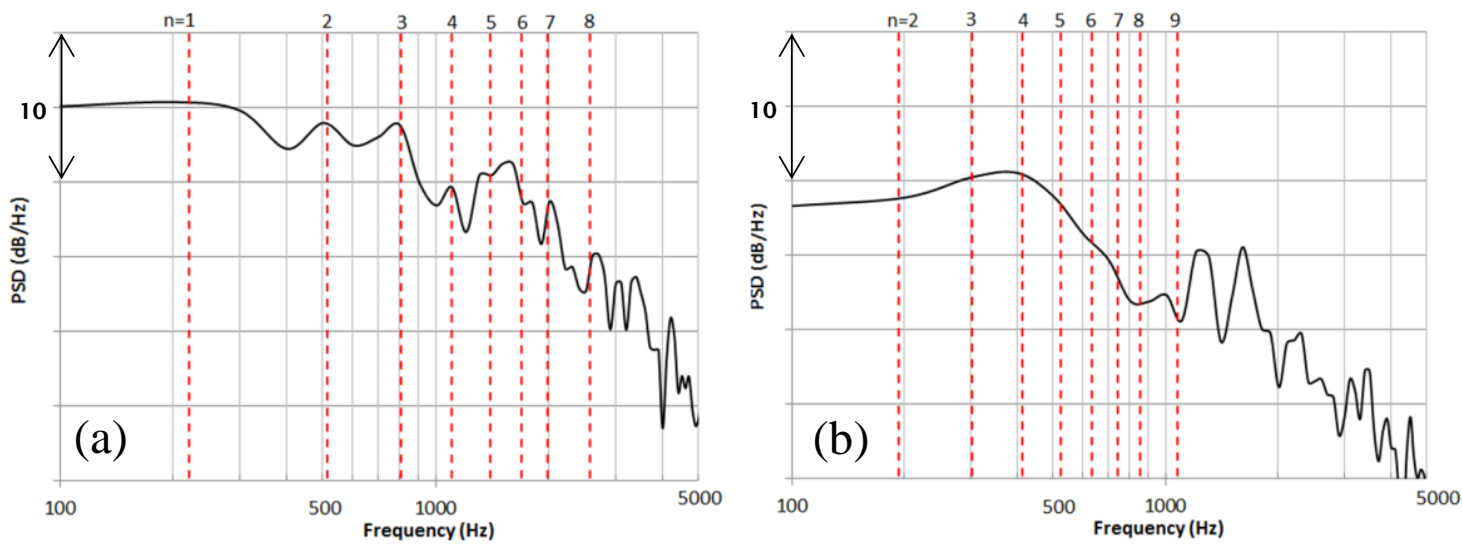

Figure 24: PSD plot (pressure) at cavity trailing edge compared with Rossiter modes, (a) Case-2 and (b) Case-3.
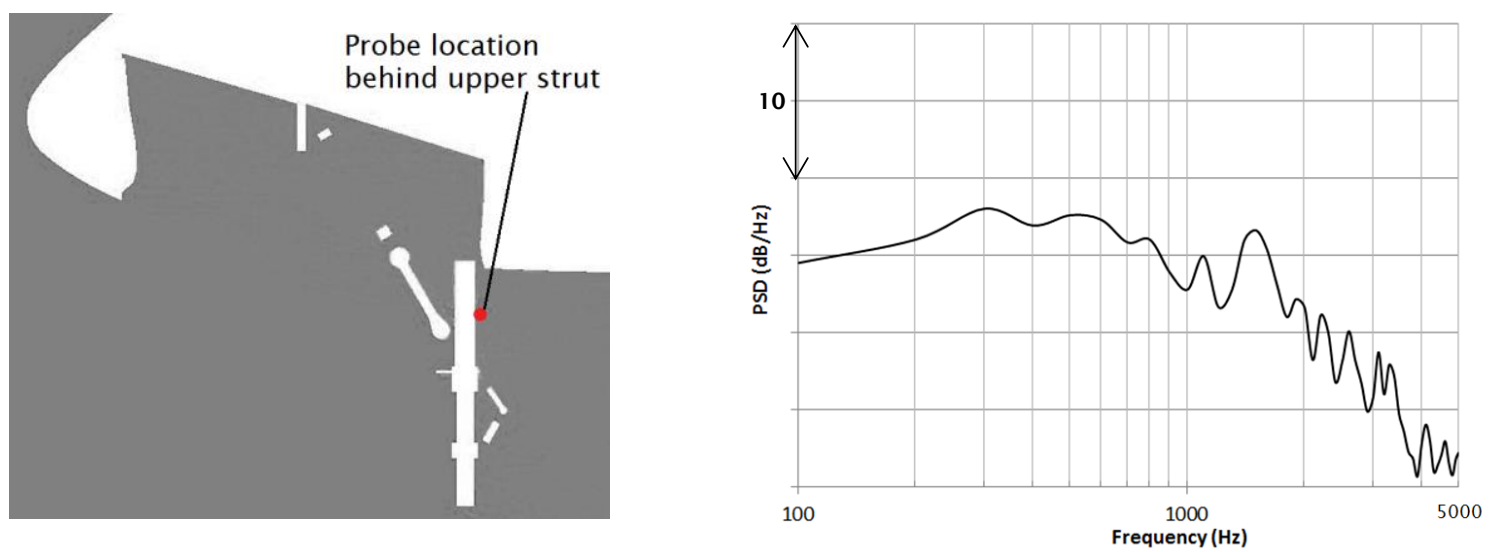

Figure 25: PSD plot (pressure) for probe behind the upper strut (Case-3). 